This is the peer reviewed version of the following article: Synlett 2010, 881, which has been published in

final form at https://www.thieme-connect.com/ejournals/abstract/synlett/doi/10.1055/s-0029-1219543. Copyright

\title{
Synthesis of Chiral Bifunctional (Thio)Urea $N$-Heterocyclic Carbenes
}

\author{
Jonathan P. Brand, José Ignacio Osuna Siles and Jérôme Waser* \\ Laboratory of Catalysis and Organic Synthesis. Ecole Polytechnique Fédérale de Lausanne EPFL SB ISIC LCSO, BCH 4306, 1015 \\ Lausanne $(\mathrm{CH})$. \\ Fax: (+) 41216939700 . \\ E-mail: jerome.waser@epfl.ch.
}

Received: The date will be inserted once the manuscript is accepted.

\begin{abstract}
The rapid and modular synthesis of the first bifunctional $\mathrm{N}$-heterocyclic carbenes bearing a (thio)urea moiety as $\mathrm{H}$-bond donor group was reported. Different analogues could be accessed in 7 steps from cheap $(S)$-pyroglutamic acid in good overall yields (14 to $30 \%$ ). The synthesized carbenes were active catalysts in the benzoin reaction.
\end{abstract}

Key words: Umpolung, asymmetric catalysis, carbenes, homogeneous catalysis, chiral pool.

The inversion of the inherent reactivity of molecules (Umpolung) is a powerful tool to increase the flexibility of complex molecule synthesis. ${ }^{1}$ Nature uses $N$ heterocyclic carbene (NHC) for catalytic Umpolung through the coenzyme thiamine. ${ }^{2}$ Synthetic $N$ heterocyclic carbenes have been highly efficient for catalytic asymmetric Umpolung reactions. ${ }^{3}$ Despite the exploding interest in this field, intermolecular crosscondensation reactions remain challenging due to the competitive self-condensation of aldehydes (the benzoin reaction). For example, the asymmetric addition of acyl anion equivalents generated from $N$-heterocyclic carbenes and aldehydes on imines or Michael acceptors (the Stetter reaction) is still a difficult process, with only few successful reports. ${ }^{4}$

A selective activation of the electrophile could circumvent this limitation. The use of H-donor has been especially efficient for organocatalytic electrophilic activation. ${ }^{5}$ Further development showed that the use of bifunctional catalysts can improve intermolecular processes through the activation of both reaction partners. ${ }^{6}$

In 2008 , Ye reported a bifunctional $N$-heterocyclic carbene catalyst $1 \mathrm{a}$ bearing an alcohol as $\mathrm{H}$-bond donors for aza-Baylis-Hillman reactions (Figure 1). ${ }^{7}$ Recently, Connon reported a bifunctional catalyst $\mathbf{2}$ based on an aminoindanol scaffold using an amide as H-bond donor. ${ }^{8}$ Connon and Zeitler then showed that catalyst $\mathbf{1 b}$ bearing a pentafluorophenyl group on the triazolium ring was highly efficient in the asymmetric benzoin reaction. ${ }^{9}$ These first reports showed the potential of combining $\mathrm{H}$ bonding with $N$-heterocyclic carbenes. Unfortunately, either yields and enantioselectivities were only moderate or the scope was limited to homo-condensation. The design of alternative structures is consequently required to develop more efficient and selective heterocondensation reactions.

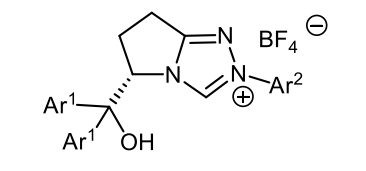

1a: $\mathrm{Ar}^{1}=3,5-\left(\mathrm{CF}_{3}\right)-\mathrm{C}_{6} \mathrm{H}_{3} ; \mathrm{Ar}^{2}=\mathrm{C}_{6} \mathrm{H}_{5}$ 1b: $\mathrm{Ar}^{1}=\mathrm{C}_{6} \mathrm{H}_{5} ; A r^{2}=\mathrm{C}_{6} \mathrm{~F}_{5}$

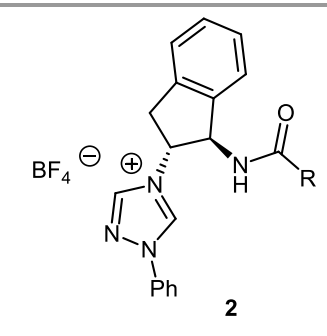

Figure 1 Bifunctional catalysts designed by Ye and Connon

Surprisingly, (thio)ureas, which have been highly successful as H-bond donors in catalysis, have never been used in combination with $N$-heterocyclic carbenes for bifunctional activation. However, Scheidt reported a combination of urea catalysis together with the stoichiometric use of $N$-heterocyclic carbenes for the conjugate addition of preformed thiazolium carbinols to nitroalkenes. ${ }^{10}$ We wish here to report a straightforward and modular synthesis of bifunctional $N$-heterocyclic carbene (thio)urea precatalysts as well as their primary evaluation in the benzoin reaction.

The main concern in the design of bifunctional $\mathrm{N}$ heterocyclic carbene catalysts is the possible intramolecular self-quenching due to the presence of both the basic nucleophilic carbene and the acidic proton of the (thio)urea. To avoid this pitfall, triazolium precursors were chosen, as they lead to less basic $N$-heterocyclic carbene catalysts. Furthermore, the substituents on both the triazolium and the (thio)urea would allow tuning the acid-base properties. Since 13 years, numerous triazolium scaffolds have been successful. ${ }^{3}$ Among them, the bicyclic structure based on pyroglutamic acid introduced by Leeper ${ }^{3 b}$ particularly attracted our interest, as it would allow a cheap and rapid access to the desired thio(urea) bifunctional catalysts 3 (Scheme 1).

A late stage introduction of diversity for both the triazolium and (thio)urea substituents is desirable in the synthesis, as these groups will play a crucial role for activity and stereoselectivity. Two strategies can be envisioned (Scheme 1). The precatalyst 3 can be obtained from the corresponding lactam $\mathbf{4}$. Lactam $\mathbf{4}$ can be accessed from azide $\mathbf{5}$, which is easily synthesized from pyroglutamic acid 6 (Route A). ${ }^{11}$ Alternatively, precatalyst $\mathbf{3}$ can be obtained from the corresponding azide $\mathbf{7}$ by reduction and (thio)urea introduction. The triazolium will be formed from azidolactam 5 (Route B). The challenge of route $\mathbf{A}$ is to form the triazolium ring in pres- 
ence of the (thio)urea group. The main challenges of route $\mathbf{B}$ are the reduction of the azide $\mathbf{7}$ in presence of the charged triazolium ring as well as the stability of the amine intermediate formed after reduction of the azide, which could attack intramolecularly the triazolium ring.

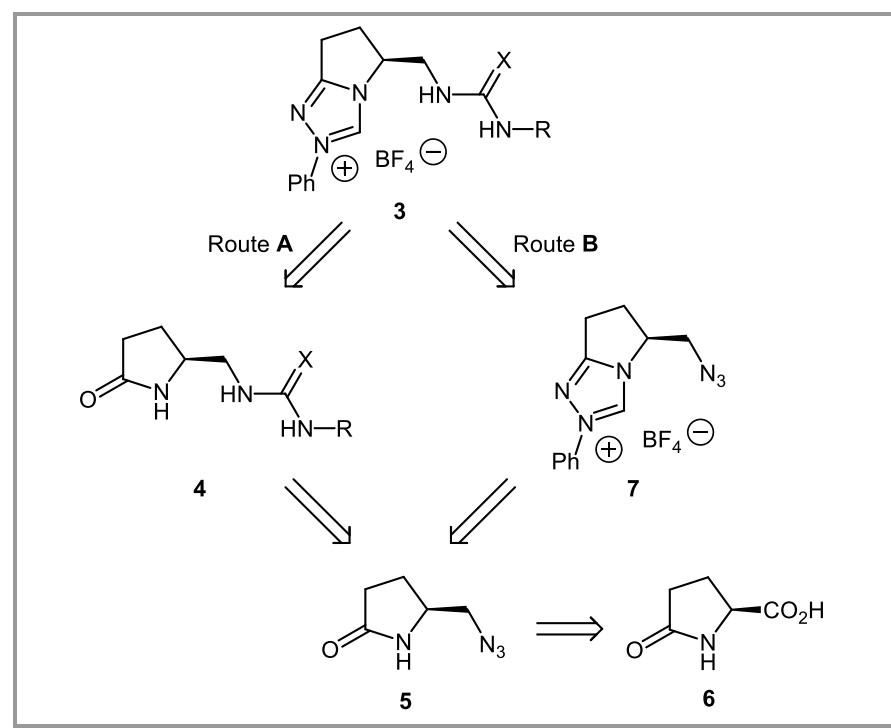

Scheme 1 Retrosynthetic analysis for triazolium-(thio)ureas $\mathbf{3}$

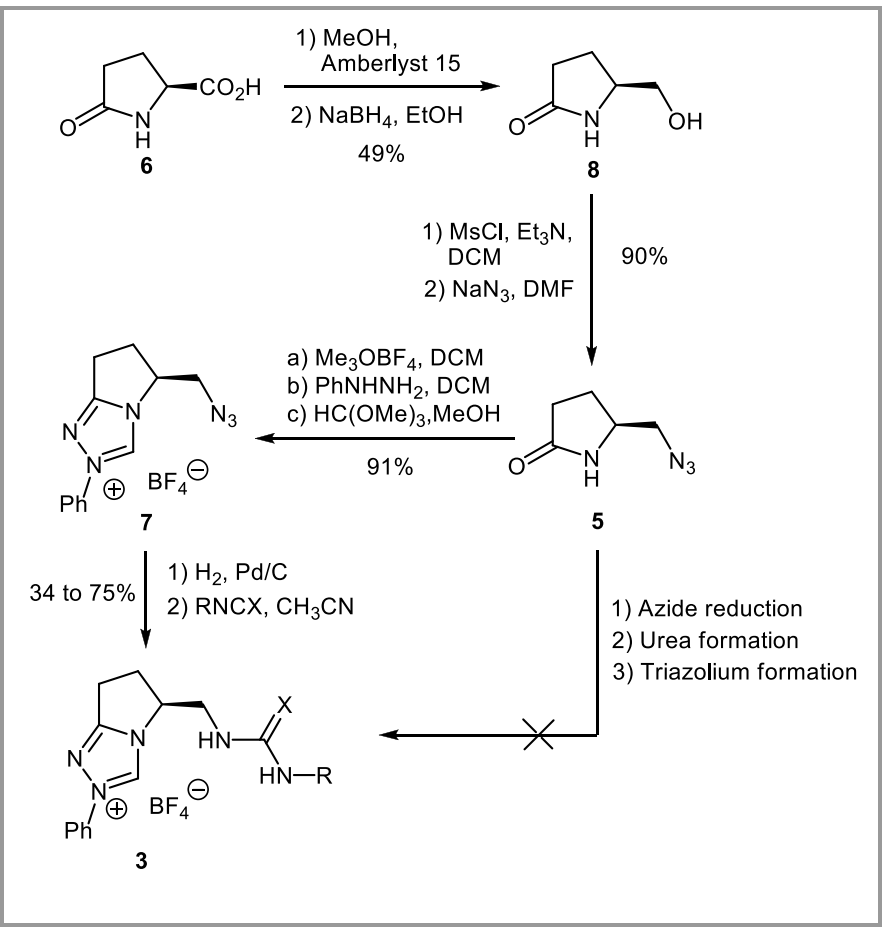

Scheme 2 Synthesis of triazolium-thio(ureas) 3

$(S)$-Pyroglutamic acid $\mathbf{6}$ was first converted in alcohol 8 via an esterification - reduction sequence on a $13 \mathrm{~g}$ scale without column chromatography (Scheme 2). ${ }^{11}$ The alcohol was then mesylated and converted to azide $\mathbf{5}$ in high yield using a slightly modified reported procedure..$^{11}$ As preliminary investigations for the conversion of $\mathbf{5}$ into $\mathbf{3}$ using route $\mathbf{A}$ were not promising, we turned to synthesis strategy B. Triazolium $\mathbf{7}$ was formed in high yield using a procedure reported by Rovis. ${ }^{12}$ A single crystallization was sufficient for purification. Azide 7 was then reduced using $\mathrm{Pd} / \mathrm{C}$ under hydrogen during one hour. Prolonged exposition to the reaction conditions led to the hydrogenation of the triazolium ring. Moreover, formation of the iminophosphorane intermediate with triphenylphosphine and direct transformation with phenylisocyanate into the urea was unsuccessful. ${ }^{13}$ Noteworthy, the reduction of the azide failed if the triazolium was substituted with a pentafluorophenyl group. This was probably due to both triazolium ring hydrogenation as well as intramolecular attack of the amine on the triazolium, as both processes are expected to be accelerated in the presence of electron-withdrawing groups. Fortunately, these side-reactions were not observed with 7 . Triazolium-(thio)ureas $\mathbf{3}$ were finally obtained by reaction of the amines with the corresponding (thio)isocyanates. This last step allowed the easy generation of analogues (Figure 2).

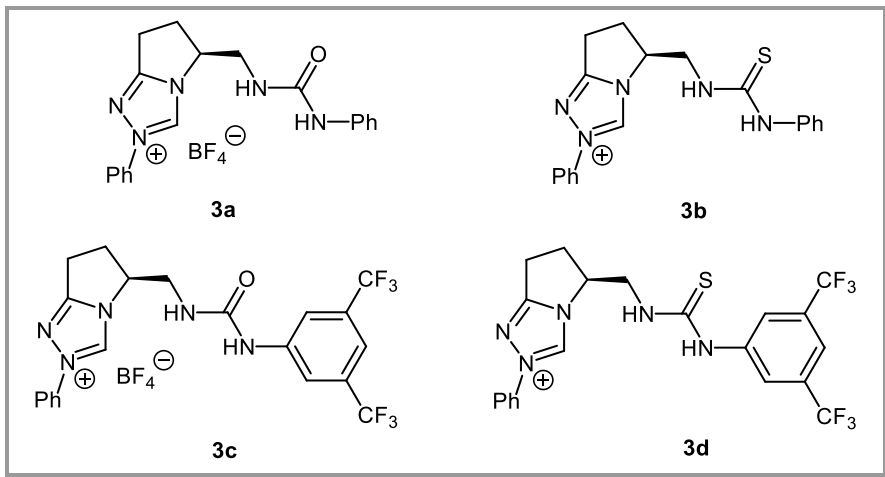

Figure 2 Different triazolium-(thio)ureas synthesized

Once the precatalysts in hand, they were evaluated in the benzoin reaction. Activity in this reaction would show that the catalysts are not inhibited by intramolecular selfquenching. To our delight, benzoin product 10 was obtained when using triazolium 3a with potassium carbonate in THF. On the other hand, running the reaction in toluene or dichloromethane did not afford any product. Triazolium-(thio)ureas 3a-c gave good yields but only moderate enantioselectivities. Triazolium-thiourea 3d gave benzoin product in low yield but very good enantioselectivity. Although the obtained yields and selectivities still need further improvement to reach the level of the best catalysts reported for asymmetric benzoin reactions, the observed activity constitutes an important proof of concept that a $N$-heterocyclic carbene and a (thio)urea can be combined in a single catalyst.

In this letter, we have reported the straightforward synthesis of (thio)urea bifunctional $N$-heterocyclic catalysts starting from cheap chiral pyroglutamic acid 6 . The main features of the synthesis were the triazolium ring formation in presence of the azide functionality and the selective reduction of the azide over the triazolium ring. The synthesis included a late stage introduction of diversity which allowed an easy access to diverse analogues. Four different triazolium (thio)ureas were synthesized in good overall yield (14\% to 30\%) over seven steps from $(S)$-pyroglutamic acid 6. Alternatively the precatalysts 
can be obtained from commercially available $(S)-5$ (hydroxymethyl)pyrrolidin-2-one $\mathbf{8}$ in five steps (overall yield: 28 to $61 \%$ ).

Table 1 Evaluation of triazolium-(thio)urea $\mathbf{3 a - 3 d}$ in the benzoin reaction

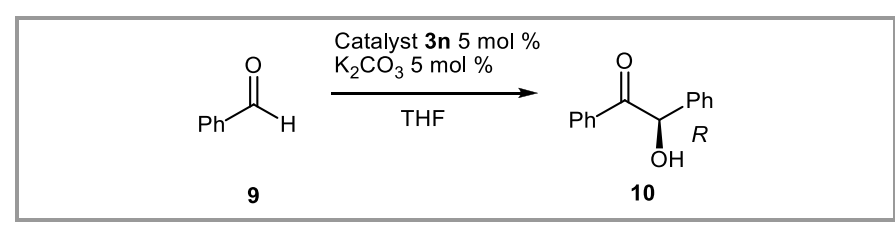

\begin{tabular}{llll}
\hline Entry & Precatalyst & Yield (\%) $^{\mathrm{a}}$ & Ee (\%) \\
\hline 1 & 3a & 47 & 64 \\
2 & 3b & 81 & 60 \\
3 & 3c & 85 & 42 \\
4 & 3d & 17 & 90 \\
\hline
\end{tabular}

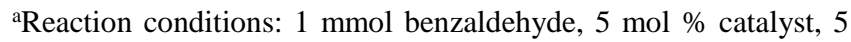
mol $\% \mathrm{~K}_{2} \mathrm{CO}_{3}$ in $\mathrm{THF}(1 \mathrm{~mL})$ at $23^{\circ} \mathrm{C}$ for $15 \mathrm{~h}$.

The observed activity of the catalysts in the benzoin reaction demonstrated that the catalysts were stable toward intramolecular acid-base self-quenching. Thio(ureas) are known to activate electrophiles such as imines and nitroolefins stronger than simple carbonyl groups. Consequently, the synthesized catalysts would be expected to favor intermolecular reactions of aldehydes with such electrophiles over the homo-benzoin reaction. Investigations are currently undergoing toward this goal in our laboratory.

(S)-5-(Azidomethyl)-2-phenyl-6,7-dihydro-5Hpyrrolo[2,1-c] $[1,2,4]$ triazol-2-ium tetrafluoroborate (7)

$(S)$-5-(Azidomethyl)pyrrolidin-2-one (5) (1.00 g, $7.10 \mathrm{mmol}, 1$ equiv) dissolved in $\mathrm{CH}_{2} \mathrm{Cl}_{2}(5 \mathrm{ml})$ was added dropwise to a suspension of $\mathrm{Me}_{3} \mathrm{OBF}_{4}$ (1.08 g, $7.10 \mathrm{mmol}, 1$ equiv) in $\mathrm{CH}_{2} \mathrm{Cl}_{2}$ (35 $\mathrm{ml})$. After $4.5 \mathrm{~h}$, phenyl hydrazine ( $775 \mu \mathrm{l}, 7.85 \mathrm{mmol}, 1.1$ equiv) was added to the colorless solution. After $3 \mathrm{~h}$, the solution was reduced under vacuum. $\mathrm{HC}(\mathrm{OMe})_{3}(14 \mathrm{ml})$ and $\mathrm{MeOH}(2 \mathrm{ml})$ were added under air. The reaction was stirred in a sealed tube at $80^{\circ} \mathrm{C}$ for $20 \mathrm{~h}$. Once cooled, $\mathrm{MeOH}$ was added until precipitation and the resulting precipitate was collected by filtration. The mother liquors were concentrated under vacuum to half of the initial volume. The resulting precipitate was filtered. Both solids were combined and dried under vacuum to give a slightly brown solid $(1.20 \mathrm{~g})$. The mother liquors were concentrated under vacuum. The resulting oil was dissolved in $\mathrm{CH}_{3} \mathrm{CN}(10 \mathrm{ml})$ and slowly added to $\mathrm{Et}_{2} \mathrm{O}(150 \mathrm{ml})$. Filtration gave a slightly brown solid (986 $\mathrm{mg}$ ). All solids were combined, dried in high vacuum to yield 7 $(2.13 \mathrm{~g}, 6.48 \mathrm{mmol}, 91 \%)$ as a slightly brown solid. $\mathrm{R}_{\mathrm{f}} 0.4$ (DCM/MeOH, 9/1 UV). ${ }^{1} \mathrm{H}$ NMR $\left(\mathrm{CD}_{3} \mathrm{CN}\right) \delta 9.81(\mathrm{~s}, 1 \mathrm{H}, \mathrm{CH}-$ triazolium), 7.84-7.78 (m, 2H, ArH), 7.67 (m, 3H, ArH), 4.91-4.82 (m, 1H, N-CH), $4.04\left(\mathrm{dd}, J=13.2,3.8 \mathrm{~Hz}, 1 \mathrm{H}, \mathrm{CH}_{2}-\mathrm{N}_{3}\right), 3.73(\mathrm{dd}$, $\left.J=13.2,8.6 \mathrm{~Hz}, 1 \mathrm{H}, \mathrm{CH}_{2}-\mathrm{N}_{3}\right), 3.33-3.13\left(\mathrm{~m}, 2 \mathrm{H}, \mathrm{CH}_{2}\right), 3.03-2.90$ $\left(\mathrm{m}, 1 \mathrm{H}, \mathrm{CH}_{2}\right), 2.54\left(\mathrm{~m}, 1 \mathrm{H}, \mathrm{CH}_{2}\right) .{ }^{13} \mathrm{C} \mathrm{NMR}\left(\mathrm{CD}_{3} \mathrm{CN}\right) \delta 163.8$, 138.1, 136.6, 131.9, 131.2, 122.2, 61.0, 53.3, 31.0, 22.2. IR $\left(\mathrm{cm}^{-1}\right)$ : 3123(w), 2115(s), 1586(m), 1519(w), 1468(w), 1446(w), 1392(w), 1292(m), 1227(m), 1034(s), 974(m), 916(w), 875(w), 771(s), 739(w), 711(m), 689(m), 671(w). HRMS(ESI) calcd for $\mathrm{C}_{12} \mathrm{H}_{13} \mathrm{~N}_{6}+\left(\mathrm{M}-\mathrm{BF}_{4}\right)$ 241.1202, found 241.1214.

(S)-2-Phenyl-5-((3-phenylureido)methyl)-6,7-dihydro-5Hpyrrolo[2,1-c] $[1,2,4]$ triazol-2-ium tetrafluoroborate (3a)

Azide 7 (1.48 g, $4.50 \mathrm{mmol})$ was dissolved in $\mathrm{MeOH}(75 \mathrm{ml})$ under $\mathrm{N}_{2}$. Pd/C (148 mg) was then added. The flask was purged with $\mathrm{H}_{2}$. After $1 \mathrm{~h}$ under $\mathrm{H}_{2}$ (3 atm), the reaction was filtered on a minimal quantity of celite and washed with $\mathrm{MeOH}(50 \mathrm{ml})$. Solvents were removed under vacuum to give the amine $(1.34 \mathrm{~g})$ as a yellow gum which was used without further purification. ${ }^{1} \mathrm{H}$ NMR $\left(\mathrm{CD}_{3} \mathrm{CN}\right) \delta 9.88$ (s, 1H, H-triazolium), 7.85-7.76 (m, 2H, ArH), 7.71-7.60 (m, 3H, ArH), $4.72(\mathrm{~m}, 1 \mathrm{H}, \mathrm{NCH}), 3.34-3.14(\mathrm{~m}, 4 \mathrm{H}$, $\left.\mathrm{CH}_{2}+\mathrm{NCH}_{2}\right), 3.01-2.87\left(\mathrm{~m}, 2 \mathrm{H}, \mathrm{CH}_{2}\right), 2.62-2.36\left(\mathrm{~m}, 3 \mathrm{H}, \mathrm{CH}_{2}+\right.$ $\mathrm{NH}_{2}+\mathrm{H}_{2} \mathrm{O}$ ).

Phenylisocyanate $(240 \mu \mathrm{l}, 2.20 \mathrm{mmol}, 1$ equiv) was added to a stirring solution of the amine ( $667 \mathrm{mg}, 2.20 \mathrm{mmol}, 1$ equiv) in $\mathrm{CH}_{3} \mathrm{CN}(7.5 \mathrm{ml})$. The reaction was stirred at RT overnight. Solvents were removed under vacuum. The crude mixture was purified by flash column chromatography $\left(\mathrm{CH}_{2} \mathrm{Cl}_{2} / \mathrm{MeOH} 90 / 10\right)$ to give 3a (706 mg, $1.68 \mathrm{mmol}, 75 \%$ over two steps) as a slightly yellow solid. ${ }^{1} \mathrm{H}$ NMR $\left(\mathrm{CD}_{3} \mathrm{CN}\right) \delta 9.81(\mathrm{~s}, 1 \mathrm{H}, \mathrm{CH}$-triazolium), 7.79-7.74 (m, 2H, ArH), 7.66-7.61 (m, 3H, ArH), $7.45(\mathrm{~s}, 1 \mathrm{H}$, $\mathrm{NH}), 7.36$ (dd, $J=9.0,1.5 \mathrm{~Hz}, 2 \mathrm{H}, \mathrm{ArH}), 7.23$ (m, 2H, ArH), 6.97 (m, 1H, ArH), 5.77 (br t, $J=6.0 \mathrm{~Hz}, 1 \mathrm{H}, \mathrm{NH}), 4.93(\mathrm{~m}, 1 \mathrm{H}$, $\mathrm{NCH}), 3.88-3.80\left(\mathrm{~m}, 1 \mathrm{H}, \mathrm{NCH}_{2}\right), 3.52-3.41\left(\mathrm{~m}, 1 \mathrm{H}, \mathrm{NCH}_{2}\right), 3.32-$ $3.12\left(\mathrm{~m}, 2 \mathrm{H}, \mathrm{CH}_{2}\right), 3.00-2.89\left(\mathrm{~m}, 1 \mathrm{H}, \mathrm{CH}_{2}\right), 2.66-2.53(\mathrm{~m}, 1 \mathrm{H}$, $\left.\mathrm{CH}_{2}\right) .{ }^{13} \mathrm{C}$ NMR $\left(\mathrm{CD}_{3} \mathrm{CN}\right) \delta 163.0,155.7,139.7,137.5,135.7$, 130.9, 130.3, 128.8, 122.3, 121.3, 118.6, 61.5, 42.1, 30.1, 21.3. IR $\left(\mathrm{cm}^{-1}\right): 3403(\mathrm{w}), 3133$ (w), 1685 (m, 1597 (s), 1547 (s), 1500 (m), 1442 (m), 1394 (m), 1315 (w), 1229 (m), 1061 (s), 763 (s), 694 (m). HRMS(ESI) calcd for $\mathrm{C}_{19} \mathrm{H}_{20} \mathrm{~N}_{5} \mathrm{O}^{+}\left(\mathrm{M}-\mathrm{BF}_{4}\right) 334.1668$, found 334.1661 .

Supporting Information for this article is available online at http://www.thieme-connect.com/ejournals/toc/synlett.

\section{Acknowledgment}

EPFL is acknowledged for financial support.

\section{References}

(1) Seebach, D. Angew. Chem. Int. Ed. 1979, 18, 239.

(2) Mizuhara, S. ; Tamura, R. ; Arata, H. Proc. Jpn. Acad. 1951, 87, 302.

(3) (a) Enders, D.; Breuer, K.; Teles, J. H. Helv. Chim. Acta 1996, 79, 1217. (b) Knight, R. L.; Leeper, F. J. J. Chem. Soc. Perkin Trans. 1 1998, 1891. (c) Kerr, M. S.; de Alaniz, J. R.; Rovis, T. J. Am. Chem. Soc. 2002, 124, 10298. (d) Takikawa, H.; Hachisu, Y.; Bode, J. W.; Suzuki, K. Angew. Chem. Int. Ed. 2006, 45, 3492. (e) Sohn, S. S.; Rosen, E. L.; Bode, J. W. J. Am. Chem. Soc. 2004, 126, 14370. (f) Chan, A.; Scheidt, K. A. J. Am. Chem. Soc. 2007, 129, 5334. (g) For a comprehensive review see: Enders, D.; Niemeier, O.; Henseler, A. Chem. Rev. 2007, $107,5606$.

(4) (a) Mennen, S. M.; Gipson, J. D.; Kim, Y. R.; Miller, S. J. J. Am. Chem. Soc. 2005, 127, 1654. (b) Mennen, S. M.; Blank, J. T.; Tran-Dube, M. B.; Imbriglio, J. E.; Miller, S. J. Chem. Commun. 2005, 195. (c) He, M.; Bode, J. W. J. Am. Chem. Soc. 2008, 130, 418. (d) Enders, D.; Han, J. W.; Henseler, A. Chem. Commun. 2008, 3989. (e) Liu, Q.; Perreault, S.; Rovis, T. J. Am. Chem. Soc. 2008, 130, 14066. (f) DiRocco, D. A.; Oberg, K. M.; Dalton, D. M.; 
Rovis, T. J. Am. Chem. Soc. 2009, 131, 10872. (g) Liu, Q.; Rovis, T. Org. Lett. 2009, 11, 2856.

(5) (a) Sigman, M. S.; Jacobsen, E. N. J. Am. Chem. Soc. 1998 120, 4901. (b) For a comprehensive review see: Doyle, A. G.; Jacobsen, E. N. Chem. Rev. 2007, 107, 5713.

(6) For some selected examples, see: (a) Okino, T.; Hoashi, Y.; Furukawa, T.; Xu, X. N.; Takemoto, Y. J. Am. Chem. Soc. 2005, 127, 119. (b) Inokuma, T.; Hoashi, Y.; Takemoto, Y. J. Am. Chem. Soc. 2006, 128, 9413. (c) Takemoto, Y., Org. Biomol. Chem. 2005, 3, 4299. (d) Connon, S. J., Chem. Commun. 2008, 2499. (e) Berkessel, A.; Cleemann, F.; Mukherjee, S.; Muller, T. N.; Lex, J. Angew. Chem. Int. $E d$. 2005, 44, 807. (f) Wu, F.-H.; Ran, H.; Khan, J.; Deng, L. Angew. Chem. Int. Ed. 2006, 45, 4301.

(7) He, L.; Zhang, Y. R.; Huang, X. L.; Ye, S. Synthesis 2008 , 2825.

(8) O'Toole, S. E.; Connon, S. J. Org. Biomol. Chem. 2009, 7 , 3584.

(9) Baragwanath, L.; Rose, C. A.; Zeitler, K.; Connon, S.J. J. Org. Chem. 2009, 74, 9214.

(10) Mattson, A. E.; Zuhl, A. M.; Reynolds, T. E.; Scheidt, K. A. J. Am. Chem. Soc. 2006, 128, 4932.

(11) Bateman, L.; Breeden, S. W.; O'Leary, P., Tetrahedron Asymmetry 2008, 19, 391.

(12) Kerr, M. S.; de Alaniz, J. R.; Rovis, T. J. Org. Chem. 2005, 70, 5725.

(13) Bianchi, A.; Ferrario, D.; Bernardi, A. Carbohydrate Res. 2006, 341, 1438. 
Chiral Bifunctional (Thio)Urea $N$-Heterocyclic Carbenes
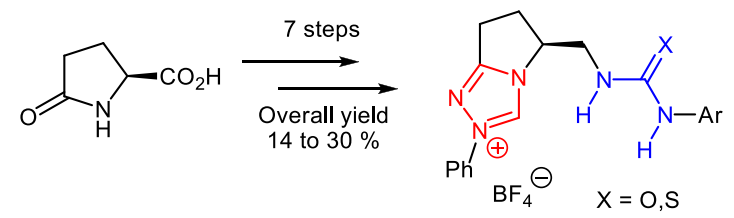


\section{Supporting information}

\section{Synthesis of Chiral Bifunctional (Thio)Urea $N$-Heterocyclic Carbenes \\ Jonathan P. Brand, José Ignacio Osuna Siles and Jérôme Waser * \\ Laboratory of Catalysis and Organic Synthesis, Ecole Polytechnique Fédérale de Lausanne, EPFL SB ISIC LCSO, BCH 4306, 1015 Lausanne, Switzerland}

13 pages

1. General Methods $\quad$ S2

2. (Thio)Urea $N$-Heterocyclic Carbenes Synthesis $\quad \mathrm{S} 3$

$\begin{array}{ll}\text { 3. Benzoin Reaction } & \text { S7 }\end{array}$

4. Spectra of New compounds $\quad$ S8 


\section{General Methods}

All reactions were carried out in oven dried glassware under an atmosphere of nitrogen, unless stated otherwise. For quantitative flash chromatography technical grade solvents were used. For flash chromatography for analysis, HPLC grade solvents from Sigma-Aldrich were used. THF, $\mathrm{Et}_{2} \mathrm{O}, \mathrm{CH}_{3} \mathrm{CN}_{\text {, }}$ toluene, hexane and $\mathrm{CH}_{2} \mathrm{Cl}_{2}$ were dried by passage over activated alumina under nitrogen atmosphere $\left(\mathrm{H}_{2} \mathrm{O}\right.$ content $<10 \mathrm{ppm}$, Karl-Fischer titration). All chemicals and solvents were purchased from Acros, Aldrich, Fluka, VWR, Aplichem or Merck and used as such unless stated otherwise. Chromatographic purification was performed as flash chromatography using Macherey-Nagel silica 40-63, $60 \AA$, using the solvents indicated as eluent with 0.1-0.5 bar pressure. TLC was performed on Merck silica gel $60 \mathrm{~F}_{254}$ TLC glass plates or aluminium plates and visualized with UV light, permanganate stain, CAN stain, Ninhydrine or Anisaldehyde stain. Melting points were measured on a Büchi B-540 melting point apparatus using open glass capillaries, the data is uncorrected. ${ }^{1} \mathrm{H}-\mathrm{NMR}$ spectra were recorded on a Brucker DPX-400 $400 \mathrm{MHz}$ spectrometer in chloroform-d, $\mathrm{CD}_{3} \mathrm{CN}$, DMSO- $\mathrm{d}_{6}$ or $\mathrm{CD}_{3} \mathrm{OD}$, all signals are reported in ppm with the internal chloroform signal at $7.26 \mathrm{ppm}$, the internal $\mathrm{CD}_{3} \mathrm{CN}$ signal at $1.94 \mathrm{ppm}$, the internal DMSO signal at $2.50 \mathrm{ppm}$ or the internal methanol signal at $3.30 \mathrm{ppm}$ as standard. The data is being reported as $(\mathrm{s}=$ singlet, $\mathrm{d}=$ doublet, $\mathrm{t}=$ triplet, $\mathrm{q}=$ quadruplet, $\mathrm{q} \mathrm{i}=$ quintet, $\mathrm{m}=$ multiplet or unresolved, $\mathrm{br}=$ broad signal, app $=$ apparent, coupling constant(s) in $\mathrm{Hz}$, integration; interpretation). ${ }^{13} \mathrm{C}-\mathrm{NMR}$ spectra were recorded with ${ }^{1} \mathrm{H}$-decoupling on a Brucker DPX-400 $100 \mathrm{MHz}$ spectrometer in chloroform-d, $\mathrm{CD}_{3} \mathrm{CN}$, DMSO- $\mathrm{d}_{6}$ or $\mathrm{CD}_{3} \mathrm{OD}$, all signals are reported in ppm with the internal chloroform signal at $77.0 \mathrm{ppm}$, the internal $\mathrm{CD}_{3} \mathrm{CN}$ signal at $118.3 \mathrm{ppm}$, the internal DMSO signal at $39.5 \mathrm{ppm}$ or the internal methanol signal at $49.0 \mathrm{ppm}$ as standard. Infrared spectra were recorded on a JASCO FT-IR B4100 spectrophotometer with an ATR PRO410-S and a ZnSe prisma and are reported as $\mathrm{cm}^{-1}$ ( $\mathrm{w}=$ weak, $\mathrm{m}=$ medium, $\mathrm{s}=$ strong, br $=$ broad). Gas chromatographic and low resolution mass spectrometric measurements were performed on a PerkinElmer Clarus 600 gas chromatographer and mass spectrometer using a Perkin-Elmer Elite fused silica column (length: $30 \mathrm{~m}$, diameter: $0.32 \mathrm{~mm}$ ) and Helium as carrier gas. High resolution mass spectrometric measurements were performed by the mass spectrometry service of ISIC at the EPFL on a MICROMASS (ESI) Q-TOF Ultima API. HPLC measurement were done on a JASCO HPLC system with an AS2055 Autosampler, a PU 2089 Pump, a UV 2075 detector and a SEDEX 85 (SEDERE) detector using a CHIRALPAK IC column from DAICEL Chemical Industries Ltd. HPLC grade solvents from SigmaAldrich were used. 


\section{II. (Thio)Urea $N$-Heterocyclic Carbenes Synthesis}

(S)-Methyl 5-oxopyrrolidine-2-carboxylate (11)

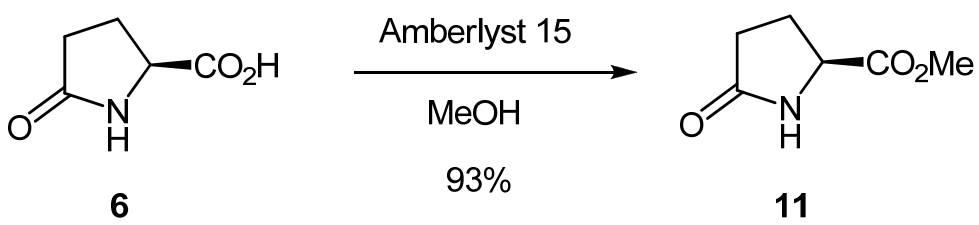

Following the reported method, ${ }^{1}$ Amberlyst 15 (11.4 g) was added to a suspension of $(S)$-(-)-Pyrrolidone5-carboxilic acid (6) $(30.0 \mathrm{~g}, 0.230 \mathrm{~mol})$ in $\mathrm{MeOH}(150 \mathrm{~mL})$ under air. The mixture was stirred overnight at reflux. After cooling, the mixture was filtered, the resin washed with $\mathrm{MeOH}$ and the solution was concentrated under vacuum to yield 11 (30.8 g, 0.215 mol, 93\%) as a yellow oil. $\mathrm{R}_{\mathrm{f}} 0.28$ (EtOAc, $\left.\mathrm{KMnO}_{4}\right)$. ${ }^{1} \mathrm{H}$ NMR $\left(\mathrm{CDCl}_{3}\right) \delta$ 7.12-6.62 (br m, $\left.1 \mathrm{H}, \mathrm{NH}\right), 4.25(\mathrm{~m}, 1 \mathrm{H}, \mathrm{CH}), 3.74\left(\mathrm{~s}, 3 \mathrm{H}, \mathrm{CH}_{3}\right), 2.55-2.12(\mathrm{~m}, 4 \mathrm{H}$, $\left.\mathrm{CH}_{2}\right) .{ }^{1} \mathrm{H}$ NMR spectra corresponded to the literature values. ${ }^{1}$

\section{(S)-5-(Hydroxymethyl)pyrrolidin-2-one (8)}

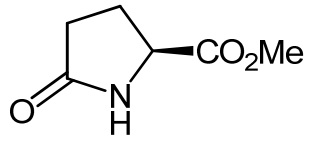

11

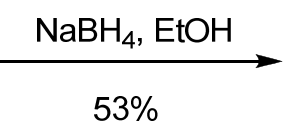

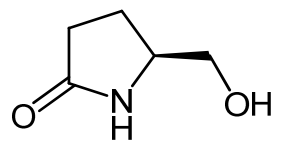

8

$\mathrm{NaBH}_{4}$ (8.13 g, 0.215 mol, 1 equiv) was added portionwise to a stirring solution of the ester $\mathbf{1 1}$ (30.8 g, $0.215 \mathrm{~mol}, 1$ equiv) in $\mathrm{EtOH}(250 \mathrm{ml})$ at $0^{\circ} \mathrm{C}$. The mixture was allowed to warm up to room temperature overnight. The reaction was then quenched dropwise at $0^{\circ} \mathrm{C}$ using concentrated $\mathrm{HCl}(15 \mathrm{ml})$. After filtration and concentration under vacuum, the oil crystallized at $0^{\circ} \mathrm{C}$. The solid was then recrystallized in EtOAc $(400$ $\mathrm{ml}$ ). After hot filtration, slow cooling down up to $0^{\circ} \mathrm{C}$ lead to crystallization. The mixture was filtered; the crystals were dried under vacuum to yield $8(13.2 \mathrm{~g}, 0.115 \mathrm{~mol}, 53 \%)$ as colorless crystals. $\mathrm{R}_{\mathrm{f}} 0.70$ $\left(\mathrm{CH}_{2} \mathrm{Cl}_{2} / \mathrm{MeOH} 9 / 1, \mathrm{KMnO}_{4}\right) .{ }^{1} \mathrm{H}$ NMR $\left(\mathrm{CDCl}_{3}\right) \delta 7.41(\mathrm{~s}, 1 \mathrm{H}, \mathrm{NH}), 4.37(\mathrm{~s}, 1 \mathrm{H}, \mathrm{OH}), 3.79(\mathrm{~m}, 1 \mathrm{H}, \mathrm{CH})$, $3.66\left(\mathrm{~d}, J=11.0 \mathrm{~Hz}, 1 \mathrm{H}, \mathrm{CH}_{2} \mathrm{O}\right), 3.44\left(\mathrm{~m}, 1 \mathrm{H}, \mathrm{CH}_{2} \mathrm{O}\right), 2.42-2.26\left(\mathrm{~m}, 2 \mathrm{H}, \mathrm{CH}_{2}\right), 2.21-2.09\left(\mathrm{~m}, 1 \mathrm{H}, \mathrm{CH}_{2}\right)$, 1.85-1.71 (m, $\left.1 \mathrm{H}, \mathrm{CH}_{2}\right) .{ }^{1} \mathrm{H}$ NMR spectra corresponded to the literature values. ${ }^{1}$

(S)-(5-Oxopyrrolidin-2-yl)methyl methanesulfonate (12)<smiles>O=C1CC[C@@H](CO)N1</smiles>

8

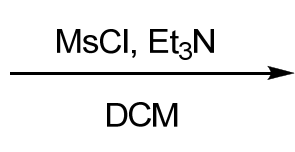

$90 \%$

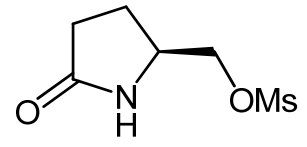

12

Following the reported procedure, ${ }^{1} \mathrm{Et}_{3} \mathrm{~N}(9.4 \mathrm{ml}, 67 \mathrm{mmol}, 2$ equiv) was added to a stirring solution of (S)-5-(hydroxymethyl)pyrrolidin-2-one (11) (4.0 g, $34 \mathrm{mmol}, 1$ equiv) in $\mathrm{CH}_{2} \mathrm{Cl}_{2}$ (45 ml) at $0^{\circ} \mathrm{C}$. $\mathrm{MsCl}(3.9$ $\mathrm{ml}, 50 \mathrm{mmol}, 1.5$ equiv) was added dropwise over $10 \mathrm{~min}$. After $1.5 \mathrm{~h}$ at $0^{\circ} \mathrm{C}$, the reaction was quenched

\footnotetext{
${ }^{1}$ Bateman, L.; Breeden, S. W.; O'Leary, P., Tetrahedron Asymmetry 2008, 19, 391.
} 
with $1 \mathrm{ml}$ of distilled water. The mixture was concentrated under vacuum. The crude mixture was purified by flash column chromatography (AcOEt/MeOH 9/1) to yield mesylate 12 (5.9 g, $30 \mathrm{mmol}, 90 \%)$ as a yellow oil. $\mathrm{R}_{\mathrm{f}} 0.30\left(\mathrm{AcOEt} / \mathrm{MeOH} 9 / 1, \mathrm{KMnO}_{4}\right) .{ }^{1} \mathrm{H} \mathrm{NMR}\left(\mathrm{CDCl}_{3}\right) \delta 6.34(\mathrm{~m}, 1 \mathrm{H}, \mathrm{NH}), 4.26(\mathrm{dd}, J=9.9$, $\left.3.5 \mathrm{~Hz}, 1 \mathrm{H}, \mathrm{CH}_{2} \mathrm{O}\right), 4.08\left(\mathrm{~m}, 1 \mathrm{H}, \mathrm{CH}_{2} \mathrm{O}\right), 4.01(\mathrm{~m}, 1 \mathrm{H}, \mathrm{CH}), 3.07\left(\mathrm{~s}, 3 \mathrm{H}, \mathrm{CH}_{3}\right), 2.48-2.23\left(\mathrm{~m}, 3 \mathrm{H}, \mathrm{CH}_{2}\right)$, 1.92-1.81 (m, $\left.1 \mathrm{H}, \mathrm{CH}_{2}\right)$.

\section{(S)-5-(Azidomethyl)pyrrolidin-2-one (5)}

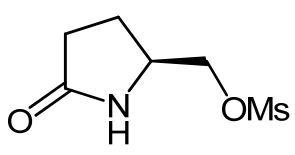

12

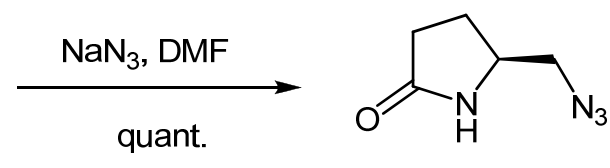

5

Following a slight modification of a reported procedure, ${ }^{1} \mathrm{NaN}_{3}(5.1 \mathrm{~g}, 78 \mathrm{mmol}, 2$ equiv) was added to a solution of (S)-(5-oxopyrrolidin-2-yl)methyl methanesulfonate (12) (7.5 g, 39 mmol, 1 equiv) in DMF (45 $\mathrm{ml})$. The reaction was stirred at $85^{\circ} \mathrm{C}$ for $15 \mathrm{~h}$. The suspension was filtered and washed with EtOAc. The mother liquor was concentrated under reduced pressure with toluene. The crude mixture was purified by flash column chromatography (AcOEt/MeOH 95/5) to yield 5 (5.6 g, $39 \mathrm{mmol}$, quant.) as a yellow oil. $\mathrm{R}_{\mathrm{f}}$ 0.51 (AcOEt/MeOH 9/1, Anisaldehyde). ${ }^{1} \mathrm{H}$ NMR $\left(\mathrm{CDCl}_{3}\right) \delta 7.57$ (s, $\left.1 \mathrm{H}, \mathrm{NH}\right), 3.79(\mathrm{~m}, 1 \mathrm{H}, \mathrm{CH}), 3.41$ (dd, $\left.J=12.0,4.5 \mathrm{~Hz}, 1 \mathrm{H}, \mathrm{CH}_{2} \mathrm{~N}_{3}\right), 3.26\left(\mathrm{dd}, J=12.0,6.0 \mathrm{~Hz}, 1 \mathrm{H}, \mathrm{CH}_{2}\right), 2.44-2.13\left(\mathrm{~m}, 3 \mathrm{H}, \mathrm{CH}_{2}\right), 1.84-1.72(\mathrm{~m}$, $\left.1 \mathrm{H}, \mathrm{CH}_{2}\right) .{ }^{13} \mathrm{C} \mathrm{NMR}\left(\mathrm{CDCl}_{3}\right) \delta 178.5,55.9,53.5,29.7,24.0 . \mathrm{IR}\left(\mathrm{cm}^{-1}\right): 3231(\mathrm{w}), 2928(\mathrm{w}), 2097(\mathrm{~s}), 1683(\mathrm{~s})$, 1462(w), 1423(w), 1264(s), 651(m). HRMS (ESI-TOF) calcd for $\mathrm{C}_{5} \mathrm{H}_{8} \mathrm{~N}_{4} \mathrm{O}^{+}(\mathrm{M}+\mathrm{H})$ 141.0776, found 141.0782. All values corresponded to the literature values. ${ }^{1}$

(S)-5-(Azidomethyl)-2-phenyl-6,7-dihydro-5H-pyrrolo[2,1-c][1,2,4]triazol-2-ium tetrafluoroborate (7)

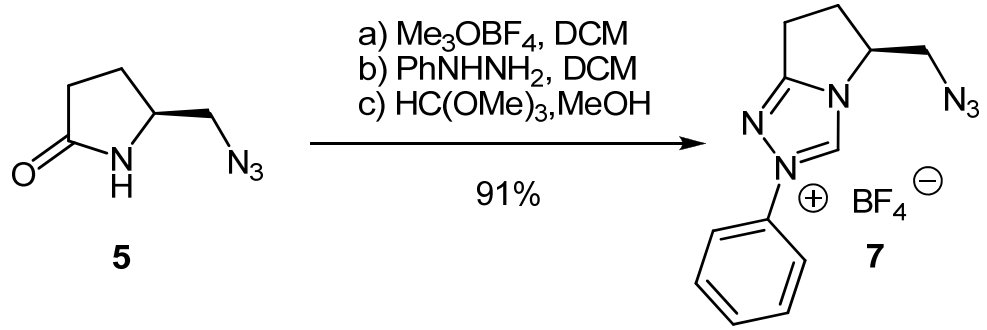

(S)-5-(Azidomethyl)pyrrolidin-2-one (5) (1.00 g, $7.10 \mathrm{mmol}, 1$ equiv) dissolved in $\mathrm{CH}_{2} \mathrm{Cl}_{2}$ (5 ml) was added dropwise to a suspension of $\mathrm{Me}_{3} \mathrm{OBF}_{4}\left(1.08 \mathrm{~g}, 7.10 \mathrm{mmol}, 1\right.$ equiv) in $\mathrm{CH}_{2} \mathrm{Cl}_{2}$ (35 ml). After $4.5 \mathrm{~h}$, phenyl hydrazine ( $775 \mu 1,7.85 \mathrm{mmol}, 1.1$ equiv) was added to the colorless solution. After $3 \mathrm{~h}$, the solution was reduced under vacuum. $\mathrm{HC}(\mathrm{OMe})_{3}(14 \mathrm{ml})$ and $\mathrm{MeOH}(2 \mathrm{ml})$ were added under air. The reaction was stirred in a sealed tube at $80^{\circ} \mathrm{C}$ for $20 \mathrm{~h}$. Once cooled, $\mathrm{MeOH}$ was added until precipitation and the resulting precipitate was collected by filtration. The mother liquors were concentrated under vacuum to half of the initial volume. The resulting precipitate was filtered. Both solids were combined and dried under vacuum to give a slightly brown solid (1.20 g,). The mother liquors were concentrated under vacuum. The resulting oil was dissolved in $\mathrm{CH}_{3} \mathrm{CN}(10 \mathrm{ml})$ and slowly added to $\mathrm{Et}_{2} \mathrm{O}(150 \mathrm{ml})$. Filtration gave a slightly brown solid $(986 \mathrm{mg})$. All solids were combined, dried in high vacuum to yield 7 (2.13 g, $6.48 \mathrm{mmol}, 91 \%)$ as a slightly brown solid. $\mathrm{R}_{\mathrm{f}} 0.4\left(\mathrm{CH}_{2} \mathrm{Cl}_{2} / \mathrm{MeOH}, 9 / 1 \mathrm{UV}\right) .{ }^{1} \mathrm{H} \mathrm{NMR}\left(\mathrm{CD}_{3} \mathrm{CN}\right) \delta 9.81$ (s, 1H, CH-triazolium), 7.84-7.78 (m, $2 \mathrm{H}, \mathrm{ArH}), 7.67$ (m, $3 \mathrm{H}, \mathrm{ArH}), 4.91-4.82(\mathrm{~m}, 1 \mathrm{H}, \mathrm{N}-\mathrm{CH}), 4.04$ (dd, $\left.J=13.2,3.8 \mathrm{~Hz}, 1 \mathrm{H}, \mathrm{CH}_{2}-\mathrm{N}_{3}\right)$, $3.73\left(\mathrm{dd}, J=13.2,8.6 \mathrm{~Hz}, 1 \mathrm{H}, \mathrm{CH}_{2}-\mathrm{N}_{3}\right), 3.33-3.13\left(\mathrm{~m}, 2 \mathrm{H}, \mathrm{CH}_{2}\right), 3.03-2.90\left(\mathrm{~m}, 1 \mathrm{H}, \mathrm{CH}_{2}\right), 2.54(\mathrm{~m}, 1 \mathrm{H}$, $\left.\mathrm{CH}_{2}\right) .{ }^{13} \mathrm{C}$ NMR $\left(\mathrm{CD}_{3} \mathrm{CN}\right) \delta 163.8,138.1,136.6,131.9,131.2,122.2,61.0,53.3,31.0,22.2 . \mathrm{IR}\left(\mathrm{cm}^{-1}\right)$ : 
3123(w), 2115(s), 1586(m), 1519(w), 1468(w), 1446(w), 1392(w), 1292(m), 1227(m), 1034(s), 974(m), 916(w), 875(w), 771(s), 739(w), 711(m), 689(m), 671(w). HRMS(ESI) calcd for $\mathrm{C}_{12} \mathrm{H}_{13} \mathrm{~N}_{6}{ }^{+}\left(\mathrm{M}-\mathrm{BF}_{4}\right)$ 241.1202, found 241.1214.

(S)-5-(Aminomethyl)-2-phenyl-6,7-dihydro-5H-pyrrolo[2,1-c][1,2,4]triazol-2-ium tetrafluoroborate (13)

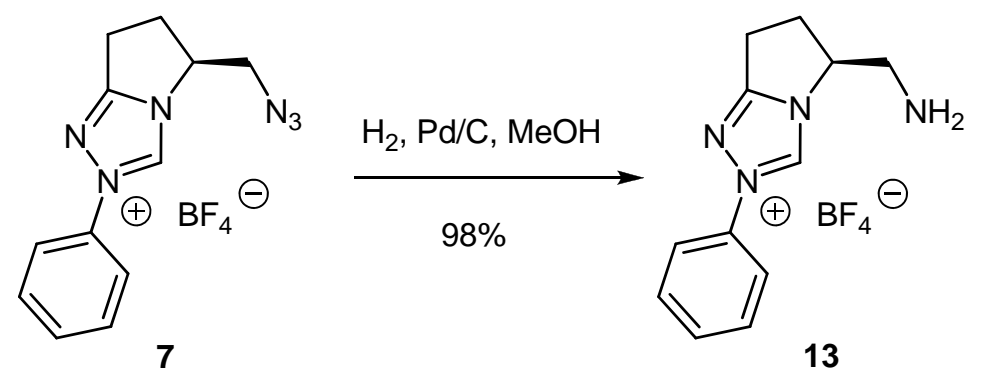

Azide 7 (1.48 g, $4.50 \mathrm{mmol})$ was dissolved in $\mathrm{MeOH}(75 \mathrm{ml})$ under $\mathrm{N}_{2}$. Pd/C (148 mg) was then added. The flask was purged with $\mathrm{H}_{2}$. After $1 \mathrm{~h}$ under $\mathrm{H}_{2}(3 \mathrm{~atm})$, the reaction was filtered on a minimal quantity of celite and washed with $\mathrm{MeOH}(50 \mathrm{ml})$. Solvents were removed under vacuum to give the amine $13(1.34 \mathrm{~g})$ as a yellow gum which was used without further purification. ${ }^{1} \mathrm{H}$ NMR $\left(\mathrm{CD}_{3} \mathrm{CN}\right) \delta 9.88(\mathrm{~s}, 1 \mathrm{H}, \mathrm{H}-$ triazolium), 7.85-7.76 (m, $2 \mathrm{H}, \mathrm{ArH}), 7.71-7.60(\mathrm{~m}, 3 \mathrm{H}, \mathrm{ArH}), 4.72(\mathrm{~m}, 1 \mathrm{H}, \mathrm{NCH}), 3.34-3.14\left(\mathrm{~m}, 4 \mathrm{H}, \mathrm{CH}_{2}\right.$ $\left.+\mathrm{NCH}_{2}\right), 3.01-2.87\left(\mathrm{~m}, 2 \mathrm{H}, \mathrm{CH}_{2}\right), 2.62-2.36\left(\mathrm{~m}, 3 \mathrm{H}, \mathrm{CH}_{2}+\mathrm{NH}_{2}+\mathrm{H}_{2} \mathrm{O}\right)$.

(S)-2-Phenyl-5-((3-phenylureido)methyl)-6,7-dihydro-5H-pyrrolo[2,1-c][1,2,4]triazol-2-ium tetrafluoroborate (3a)
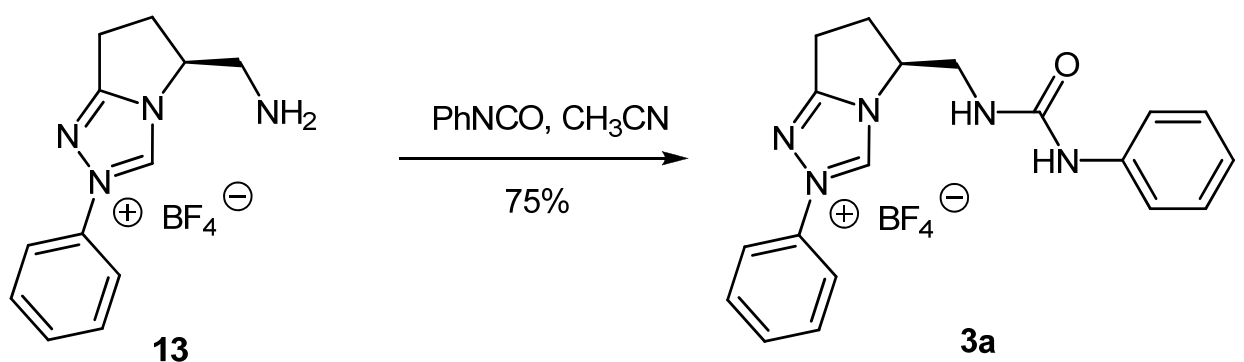

Phenylisocyanate $(240 \mu \mathrm{l}, 2.20 \mathrm{mmol}, 1$ equiv) was added to a stirring solution of the amine 13 (667 $\mathrm{mg}$, 2.20 mmol, 1 equiv) in $\mathrm{CH}_{3} \mathrm{CN}(7.5 \mathrm{ml})$. The reaction was stirred at RT overnight. Solvents were removed under vacuum. The crude mixture was purified by flash column chromatography $\left(\mathrm{CH}_{2} \mathrm{Cl}_{2} / \mathrm{MeOH} 90 / 10\right)$ to give 3a (706 mg, $1.68 \mathrm{mmol}, 75 \%$ over two steps) as a slightly yellow solid. ${ }^{1} \mathrm{H}$ NMR $\left(\mathrm{CD}_{3} \mathrm{CN}\right) \delta 9.81(\mathrm{~s}, 1$ H, CH-triazolium), 7.79-7.74 (m, 2 H, ArH), 7.66-7.61 (m, $3 \mathrm{H}, \mathrm{ArH}), 7.45$ (s, $1 \mathrm{H}, \mathrm{NH}), 7.36$ (dd, $J=9.0$, $1.5 \mathrm{~Hz}, 2 \mathrm{H}, \mathrm{ArH}), 7.23$ (m, $2 \mathrm{H}, \mathrm{ArH}), 6.97$ (m, $1 \mathrm{H}, \mathrm{ArH}), 5.77$ (br t, $J=6.0 \mathrm{~Hz}, 1 \mathrm{H}, \mathrm{NH}), 4.93$ (m, $1 \mathrm{H}$, $\mathrm{NCH}), 3.88-3.80\left(\mathrm{~m}, 1 \mathrm{H}, \mathrm{NCH}_{2}\right), 3.52-3.41\left(\mathrm{~m}, 1 \mathrm{H}, \mathrm{NCH}_{2}\right), 3.32-3.12\left(\mathrm{~m}, 2 \mathrm{H}, \mathrm{CH}_{2}\right), 3.00-2.89(\mathrm{~m}, 1 \mathrm{H}$, $\left.\mathrm{CH}_{2}\right), 2.66-2.53\left(\mathrm{~m}, 1 \mathrm{H}, \mathrm{CH}_{2}\right) .{ }^{13} \mathrm{C} \mathrm{NMR}\left(\mathrm{CD}_{3} \mathrm{CN}\right) \delta 163.0,155.7,139.7,137.5,135.7,130.9,130.3,128.8$, 122.3, 121.3, 118.6, 61.5, 42.1, 30.1, 21.3. IR ( $\left.\mathrm{cm}^{-1}\right): 3403$ (w), 3133 (w), 1685 (m, 1597 (s), 1547 (s), 1500 (m), 1442 (m), 1394 (m), 1315 (w), 1229 (m), 1061 (s), 763 (s), 694 (m). HRMS(ESI) calcd for $\mathrm{C}_{19} \mathrm{H}_{20} \mathrm{~N}_{5} \mathrm{O}^{+}$ $\left(\mathrm{M}-\mathrm{BF}_{4}\right)$ 334.1668, found 334.1661.

(S)-2-Phenyl-5-((3-phenylthioureido)methyl)-6,7-dihydro-5H-pyrrolo[2,1-c][1,2,4]triazol-2-ium tetrafluoroborate (3b) 

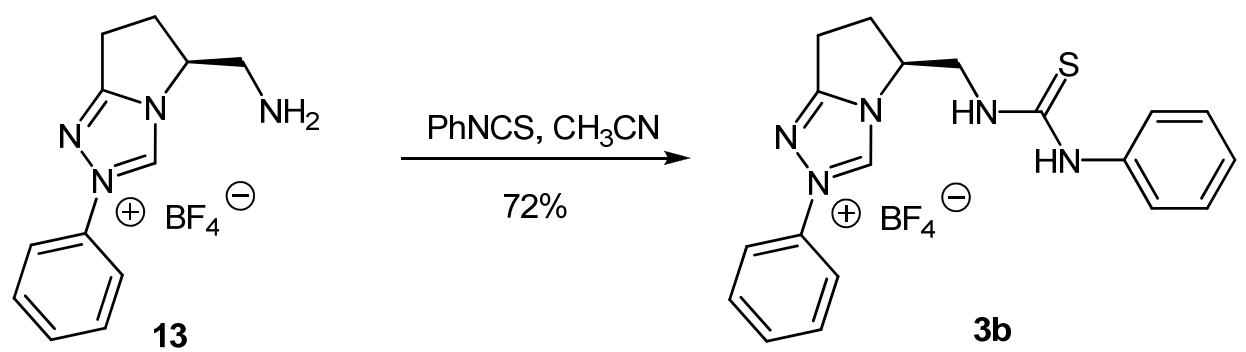

Phenylthioisocyanate ( $263 \mu 1,2.20 \mathrm{mmol}, 1$ equiv) was added to a stirring solution of amine $\mathbf{1 3}$ (667 mg, 2.20 mmol, 1 equiv) in $\mathrm{CH}_{3} \mathrm{CN}$ (7.5 ml). The reaction was stirred at RT overnight. Solvents were removed under vacuum. The crude mixture was purified by flash column chromatography $\left(\mathrm{CH}_{2} \mathrm{Cl}_{2} / \mathrm{MeOH} 90 / 10\right)$ to give $3 \mathbf{b}$ (700 mg, $1.60 \mathrm{mmol}, 72 \%$ over two steps) as a slightly yellow solid. ${ }^{1} \mathrm{H}$ NMR $\left(\mathrm{CD}_{3} \mathrm{CN}\right) \delta 9.77(\mathrm{~s}, 1$ H, CH-triazolium), 8.49 (br s, $1 \mathrm{H}, \mathrm{NH}$ ), 7.80-7.73 (m, $2 \mathrm{H}, \mathrm{ArH}), 7.68-7.61$ (m, $3 \mathrm{H}, \mathrm{ArH})$, 7.43-7.33 (m, 4 $\mathrm{H}, \mathrm{ArH}), 7.28-7.22$ (m, $1 \mathrm{H}, \mathrm{ArH}), 7.01$ (br m, $1 \mathrm{H}, \mathrm{NH}), 5.09$ (m, $1 \mathrm{H}, \mathrm{CH}-\mathrm{N}), 4.15$ (m, $1 \mathrm{H}, \mathrm{CH}-\mathrm{NH}), 4.02$ $\left(\mathrm{m}, 1 \mathrm{H}, \mathrm{CH}_{2} \mathrm{-NH}\right), 3.34-3.14\left(\mathrm{~m}, 2 \mathrm{H}, \mathrm{CH}_{2}\right), 3.01-2.88\left(\mathrm{~m}, 1 \mathrm{H}, \mathrm{CH}_{2}\right), 2.62\left(\mathrm{~m}, 1 \mathrm{H}, \mathrm{CH}_{2}\right) .{ }^{13} \mathrm{C} \mathrm{NMR}$ $\left(\mathrm{CD}_{3} \mathrm{CN}\right) \delta 183.8,163.8,138.5,138.3,136.6,131.8,131.2,130.2,127.3,126.2,122.2,61.4,47.0,31.1$, 22.0. IR ( $\left.\mathrm{cm}^{-1}\right): 3361(\mathrm{w}), 3135(\mathrm{w}), 3067(\mathrm{w}), 1592(\mathrm{~m}), 1531(\mathrm{~s}), 1498(\mathrm{~m}), 1393(\mathrm{w}), 1353(\mathrm{w}), 1316(\mathrm{~m})$, 1263(w), 1222(w), 1055(s), 764(m), 708(m), 689(m), 627(w). HRMS(ESI) calcd for $\mathrm{C}_{19} \mathrm{H}_{20} \mathrm{~N}_{5} \mathrm{O}^{+}\left(\mathrm{M}-\mathrm{BF}_{4}\right)$ 350.1440 , found 350.1443 .

(S)-5-((3-(3,5-Bis(trifluoromethyl)phenyl)ureido)methyl)-2-phenyl-6,7-dihydro-5H-pyrrolo[2,1c][1,2,4]triazol-2-ium tetrafluoroborate (3c)
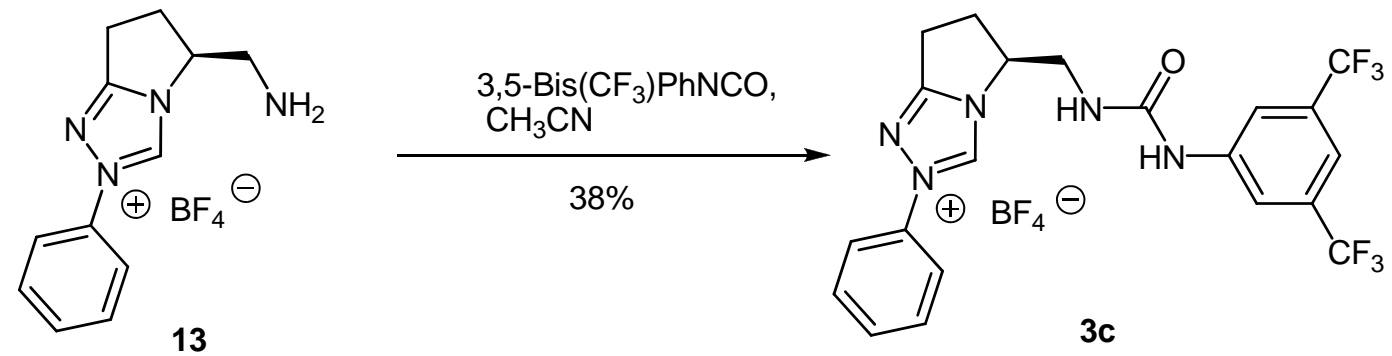

3,5-Bis $\left(\mathrm{CF}_{3}\right)$-phenylisocyanate (143 $\mu 1,0.830 \mathrm{mmol}, 1$ equiv) was added to a stirring solution of the amine 13 (250 mg, $0.830 \mathrm{mmol}, 1$ equiv) in $\mathrm{CH}_{3} \mathrm{CN}$ (2.5 ml). The reaction was stirred at RT overnight. Solvents were removed under vacuum. The resulting solid was triturated in $\mathrm{CH}_{2} \mathrm{Cl}_{2}$, filtered and solvent were removed under vacuum to give $3 \mathrm{c}(182 \mathrm{mg}, 0.330 \mathrm{mmol}, 38 \%$ over two steps $)$ as a white solid. $\mathrm{R}_{\mathrm{f}} 0.3$ $\left(\mathrm{CH}_{2} \mathrm{Cl}_{2} / \mathrm{MeOH}, 9 / 1 \mathrm{UV}\right) .{ }^{1} \mathrm{H}$ NMR $\left(\mathrm{CD}_{3} \mathrm{CN}\right) \delta 9.77$ (s, $1 \mathrm{H}, \mathrm{CH}$-triazolium), 7.97 (s, $\left.2 \mathrm{H}, \mathrm{ArH}\right), 7.89$ (s, 1 $\mathrm{H}, \operatorname{ArH}), 7.78-7.71(\mathrm{~m}, 2 \mathrm{H}, \mathrm{ArH}), 7.63-7.62$ (m, $3 \mathrm{H}, \mathrm{ArH}), 7.56$ (br s, NH), 5.92 (br t, J = 5.1 Hz, $1 \mathrm{H}$, $\mathrm{NH}), 4.96(\mathrm{~m}, 1 \mathrm{H}, \mathrm{NCH}), 3.87$ (ddd, $\left.J=15.0,5.8,3.8 \mathrm{~Hz}, 1 \mathrm{H}, \mathrm{NCH}_{2}\right), 3.51(\mathrm{dt}, J=15.0,6.7 \mathrm{~Hz}, 1 \mathrm{H}$, $\left.\mathrm{NCH}_{2}\right), 3.24\left(\mathrm{~m}, 2 \mathrm{H}, \mathrm{CH}_{2}\right), 2.96\left(\mathrm{~m}, 1 \mathrm{H}, \mathrm{CH}_{2}\right), 2.62\left(\mathrm{~m}, 1 \mathrm{H}, \mathrm{CH}_{2}\right) .{ }^{13} \mathrm{C} \mathrm{NMR}\left(\mathrm{CD}_{3} \mathrm{CN}\right) \delta 163.3,155.6$, 142.0, 137.7, 135.9, $131.4(\mathrm{q}, J=33 \mathrm{~Hz}), 131.2,130.5,123.5(\mathrm{q}, J=272 \mathrm{~Hz}), 121.5,118.0(\mathrm{q}, J=4 \mathrm{~Hz})$, $115.2(\mathrm{~m}), 61.5,42.4,30.3,21.5$. IR $\left(\mathrm{cm}^{-1}\right): 3403(\mathrm{w}), 2107(\mathrm{w}), 1703(\mathrm{w}), 1568(\mathrm{~m}), 1475(\mathrm{w}), 1443(\mathrm{w})$, 1389(m), 1279(s), 1230(w), 1183(m), 1130(s), 1062(m), 884(w), 765(w), 741(w), 704(w), 684(w). HRMS(ESI) calcd for $\mathrm{C}_{21} \mathrm{H}_{18} \mathrm{~F}_{6} \mathrm{~N}_{5} \mathrm{O}^{+}\left(\mathrm{M}-\mathrm{BF}_{4}\right) 470.1415$, found 470.1431 .

(S)-5-((3-(3,5-Bis(trifluoromethyl)phenyl)thioureido)methyl)-2-phenyl-6,7-dihydro-5H-pyrrolo[2,1c][1,2,4]triazol-2-ium tetrafluoroborate (3d) 

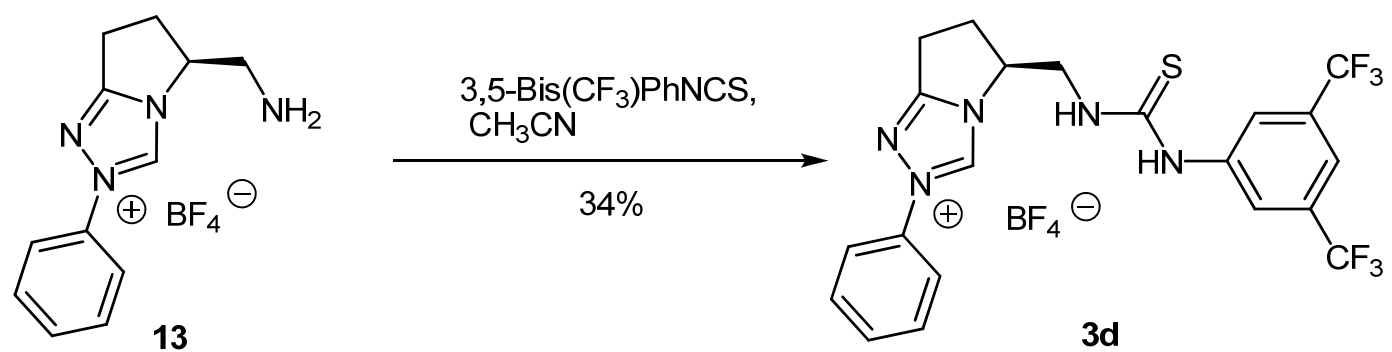

3,5-Bis $\left(\mathrm{CF}_{3}\right)$-phenylthioisocyanate $(127 \mu 1,0.700 \mathrm{mmol}, 1$ equiv.) was added to solution of the amine 13 (210 mg, $0.700 \mathrm{mmol}, 1$ equiv) in $\mathrm{CH}_{3} \mathrm{CN}(2.5 \mathrm{ml})$. The reaction was stirred at RT overnight. Solvents were removed under vacuum. The resulting solid was triturated in $\mathrm{CH}_{2} \mathrm{Cl}_{2}$, filtered and solvent were removed under vacuum to give 3d (106 mg, $0.180 \mathrm{mmol}, 26 \%$ over two steps) as a white solid. The mother liquors were concentrated under vacuum. The resulting solid was triturated in $\mathrm{CH}_{2} \mathrm{Cl}_{2}$, filtered and solvents were removed under vacuum to give $\mathbf{3 d}\left(31 \mathrm{mg}, 0.050 \mathrm{mmol}, 8 \%\right.$ over two steps) as a white solid. $\mathrm{R}_{\mathrm{f}} 0.4$ $\left(\mathrm{CH}_{2} \mathrm{Cl}_{2} / \mathrm{MeOH}, 9 / 1 \mathrm{UV}\right) .{ }^{1} \mathrm{H} \mathrm{NMR}\left(\mathrm{CD}_{3} \mathrm{CN}\right) \delta 9.80$ (s, $1 \mathrm{H}, \mathrm{CH}$-triazolium), 8.79 (br s, $\left.1 \mathrm{H}, \mathrm{NH}\right), 8.13$ (s, 2 $\mathrm{H}, \mathrm{ArH}), 7.77$ (m, $3 \mathrm{H}, \mathrm{ArH}), 7.64$ (m, $3 \mathrm{H}, \mathrm{ArH}), 7.33$ (br t, $J=5.5 \mathrm{~Hz}, 1 \mathrm{H}, \mathrm{NH}), 5.15$ (m, $1 \mathrm{H}, \mathrm{NCH}$ ), $4.19\left(\mathrm{~m}, 1 \mathrm{H}, \mathrm{NCH}_{2}\right), 4.08\left(\mathrm{~m}, 1 \mathrm{H}, \mathrm{NCH}_{2}\right), 3.27\left(\mathrm{~m}, 2 \mathrm{H}, \mathrm{CH}_{2}\right), 3.00\left(\mathrm{~m}, 1 \mathrm{H}, \mathrm{CH}_{2}\right), 2.65\left(\mathrm{~m}, 1 \mathrm{H}, \mathrm{CH}_{2}\right) .{ }^{13} \mathrm{C}$ NMR $\left(\mathrm{CD}_{3} \mathrm{CN}\right) \delta 183.0,162.9,140.6,137.2,135.5,131.2(\mathrm{q}, J=33 \mathrm{~Hz}), 130.8,130.1,124.1(\mathrm{~m}), 123.4(\mathrm{q}$, $J=271 \mathrm{~Hz}), 121.1,118.2(\mathrm{~m}), 60.2,45.8,30.1,21.0 . \mathrm{IR}\left(\mathrm{cm}^{-1}\right): 3361(\mathrm{w}), 3125(\mathrm{w}), 2114(\mathrm{w}), 1592(\mathrm{w})$, 1542(w), 1474(w), 1384(m), 1278(s), 1179(m), 1133(s), 1055(m), 888(w), 764(w), 684(w). HRMS(ESI) calcd for $\mathrm{C}_{21} \mathrm{H}_{18} \mathrm{~F}_{6} \mathrm{~N}_{5} \mathrm{~S}^{+}\left(\mathrm{M}-\mathrm{BF}_{4}\right) 486.1187$, found 486.1198 .

\section{Benzoin reaction}

General procedure: Dry $\mathrm{K}_{2} \mathrm{CO}_{3}(7 \mathrm{mg}, 0.05 \mathrm{mmol}, 0.05$ equiv) was added to precatalyst $3 \mathrm{n}$ ( $0.05 \mathrm{mmol}$, 0.05 equiv) and distilled benzaldehyde $(101 \mu 1,1.00 \mathrm{mmol}, 1$ equiv) in dry THF $(1 \mathrm{~mL})$. The reaction was sealed and stirred at $23^{\circ} \mathrm{C}$ for $15 \mathrm{~h}$. The reaction was then diluted in $\mathrm{Et}_{2} \mathrm{O}(15 \mathrm{ml})$ and water $(15 \mathrm{ml})$. After layer separation, the aqueous layer was extracted with $\mathrm{Et}_{2} \mathrm{O}(15 \mathrm{~mL})$. The organic layers were combined, washed with brine, dried over $\mathrm{MgSO}_{4}$, filtered and concentrated in vacuum. The resulting yellow oil was purified by column chromatography (PET/EtOAc, 4/1) to afford the benzoin product $\mathbf{1 0}$. $\mathrm{R}_{\mathrm{f}} 0.2$ (PET/EtOAc, 4/1 UV). ${ }^{1} \mathrm{H}$ NMR $\left(\mathrm{CDCl}_{3}\right) \delta 7.94(\mathrm{dm}, J=8.2 \mathrm{~Hz}, 2 \mathrm{H}, \mathrm{Ar} \mathrm{H}), 7.55(\mathrm{tm}, J=7.2 \mathrm{~Hz}, 1 \mathrm{H}, \mathrm{Ar}$ H), 7.42 (tm, J = 7.6 Hz, 2 H, Ar H), 7.29-7.37 (m, 5 H, Ar H), 5.98 (m, 1 H, CHO), 4.58 (br s, 1 H, OH). The ${ }^{1} \mathrm{H}$ NMR was consistent with the reported values. ${ }^{2}$

HPLC analysis: Daicel Chiralpack IC column; $20^{\circ} \mathrm{C}$; 0 to $20 \mathrm{~min}: 1 \mathrm{ml} / \mathrm{min}, 20 \mathrm{~min}$ to $30 \mathrm{~min} 1$ to 0.7 $\mathrm{ml} / \mathrm{min}$; solvent system: ${ }^{i} \mathrm{PrOH} /$ hexanes, 0 to $5 \mathrm{~min} 5 / 95,5$ to $10 \mathrm{~min} 5 / 95$ to $10 / 90,10$ to $20 \mathrm{~min} 10 / 90,20$ $\min$ to $30 \mathrm{~min} 10 / 90$ to $40 / 60$; major peak: $18.5 \mathrm{~min}$, minor peak $21.4 \mathrm{~min}$.

3a: $(50 \mathrm{mg}, 0.24 \mathrm{mmol}, 47 \%)$, ee: $64 \%$. $[\alpha]_{\mathrm{D}}{ }^{20}=-64.6(\mathrm{c}=0.8 \mathrm{in} \mathrm{MeOH})$. Consistent with reported value. ${ }^{2}$

3b: (86 mg, $0.41 \mathrm{mmol}, 81 \%)$, ee: 60\%.

3c: (90 mg, $0.14 \mathrm{mmol}, 85 \%)$, ee: 42\%.

\footnotetext{
${ }^{2}$ O'Toole, S. E.; Connon, S. J., Org. Biomol.Chem. 2009, 7, 3584.
} 
3d: (18 mg, 0.08 mmol, 17\%), ee: 90\%.

IV. Spectra of New Compounds 


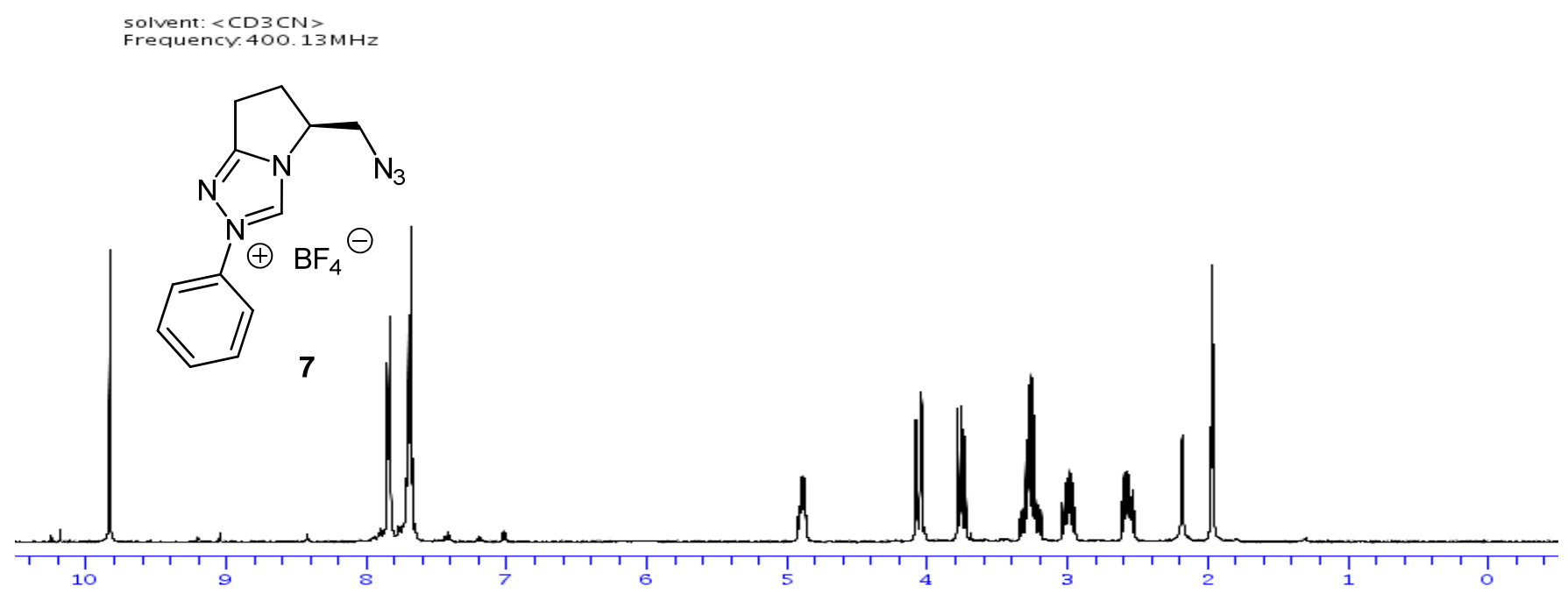

solvent: $\angle C D 3 C N>$

Frequency. $100.61 \mathrm{MHz}$
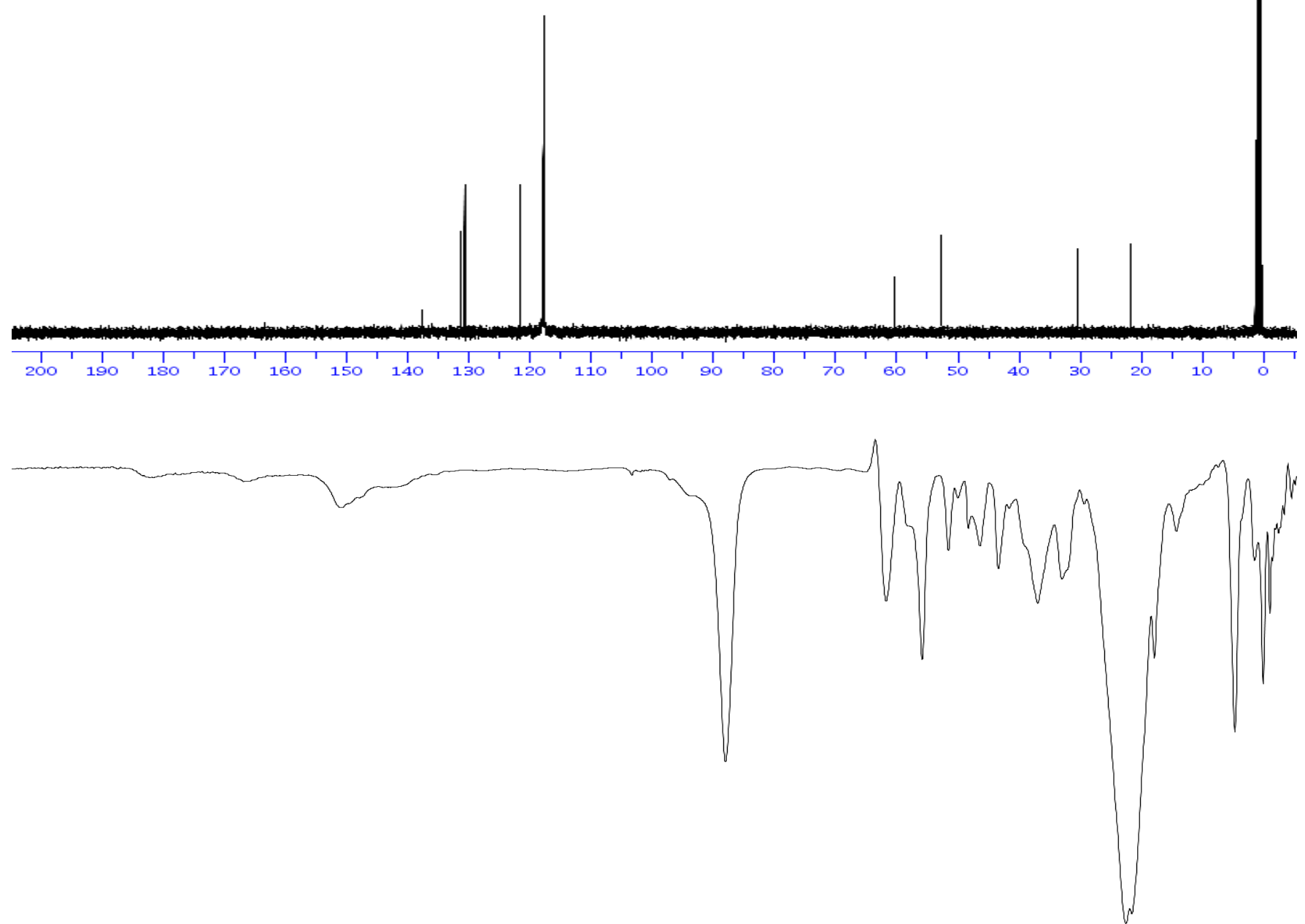

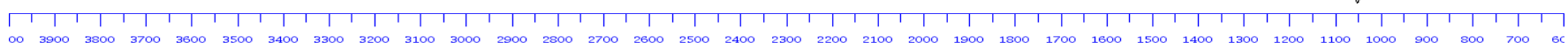

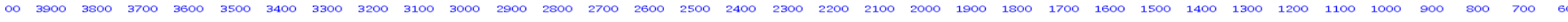



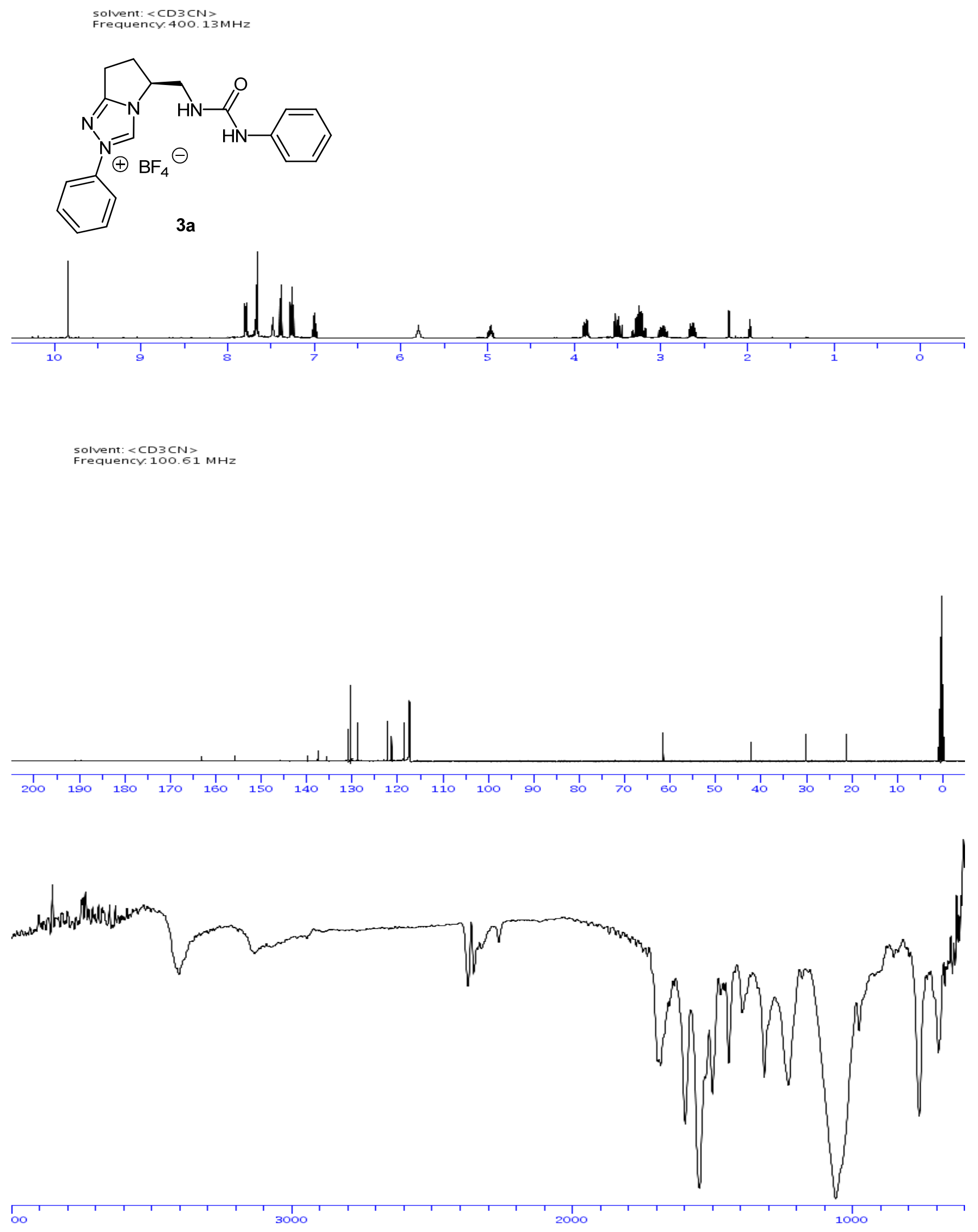
solvent: $\angle C D 3 C N>$
Frequency. $400.13 \mathrm{MHz}$

$3 b$

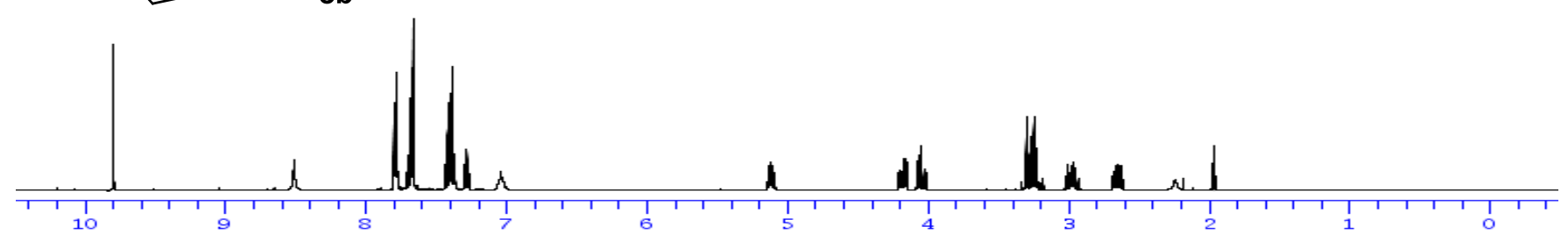

solvent: $\langle C D 3 C \mathrm{~N}\rangle$
Frequency. $100.61 \mathrm{MHz}$

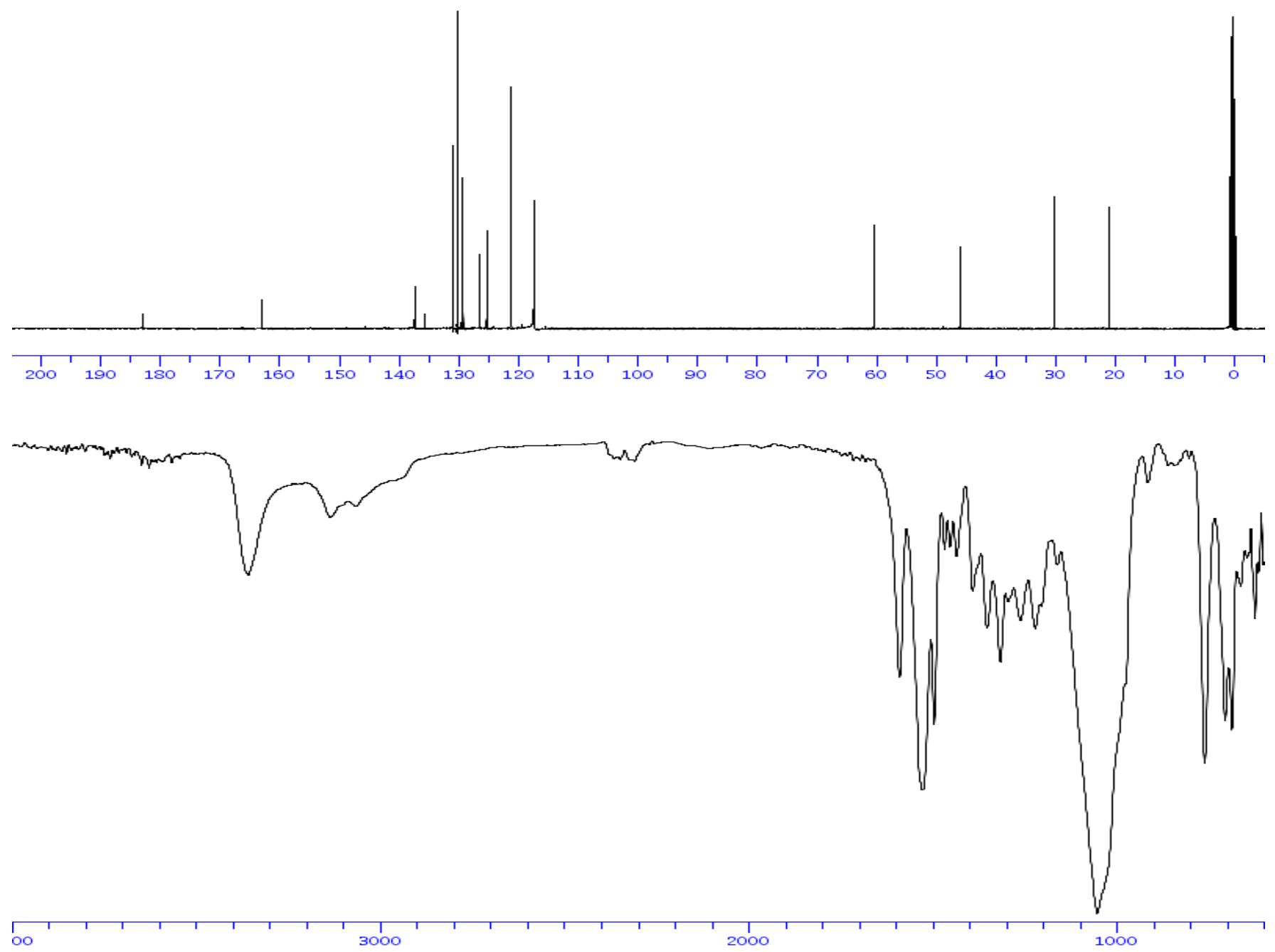

11 


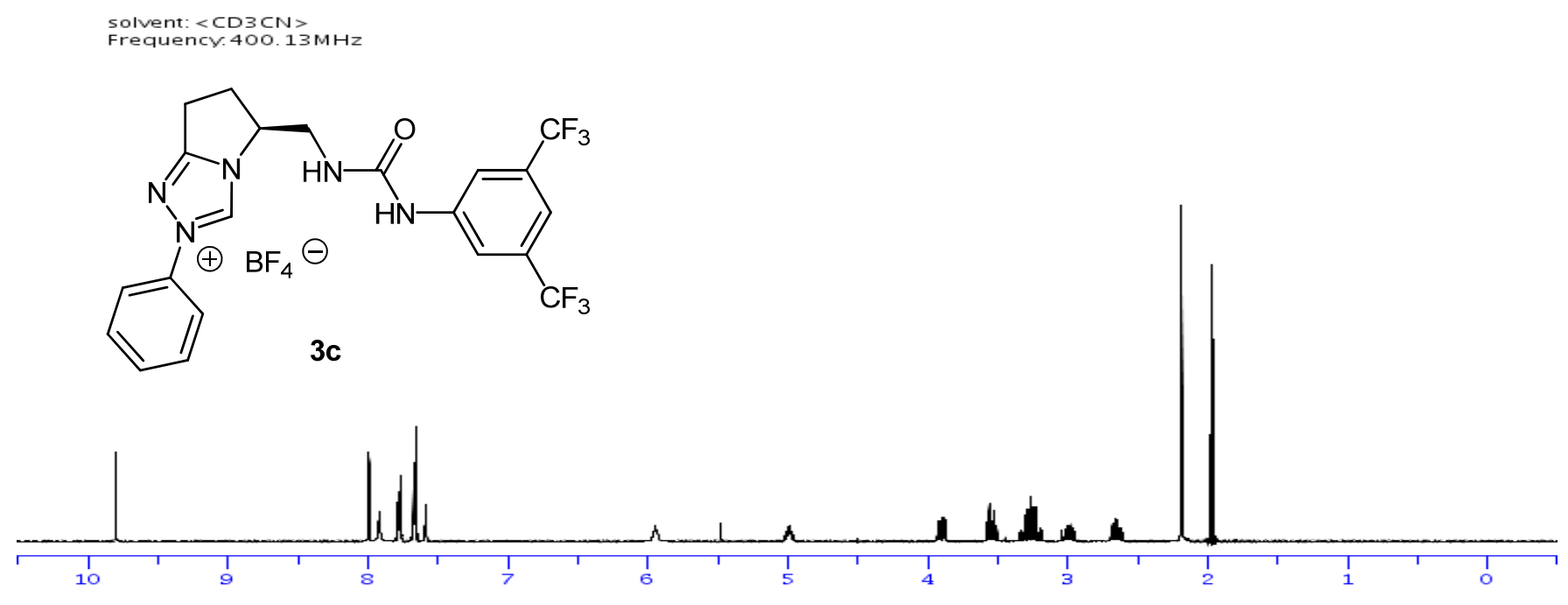

solvent: $\langle$ CD $3 C N\rangle$

Frequency.100.612769MHz
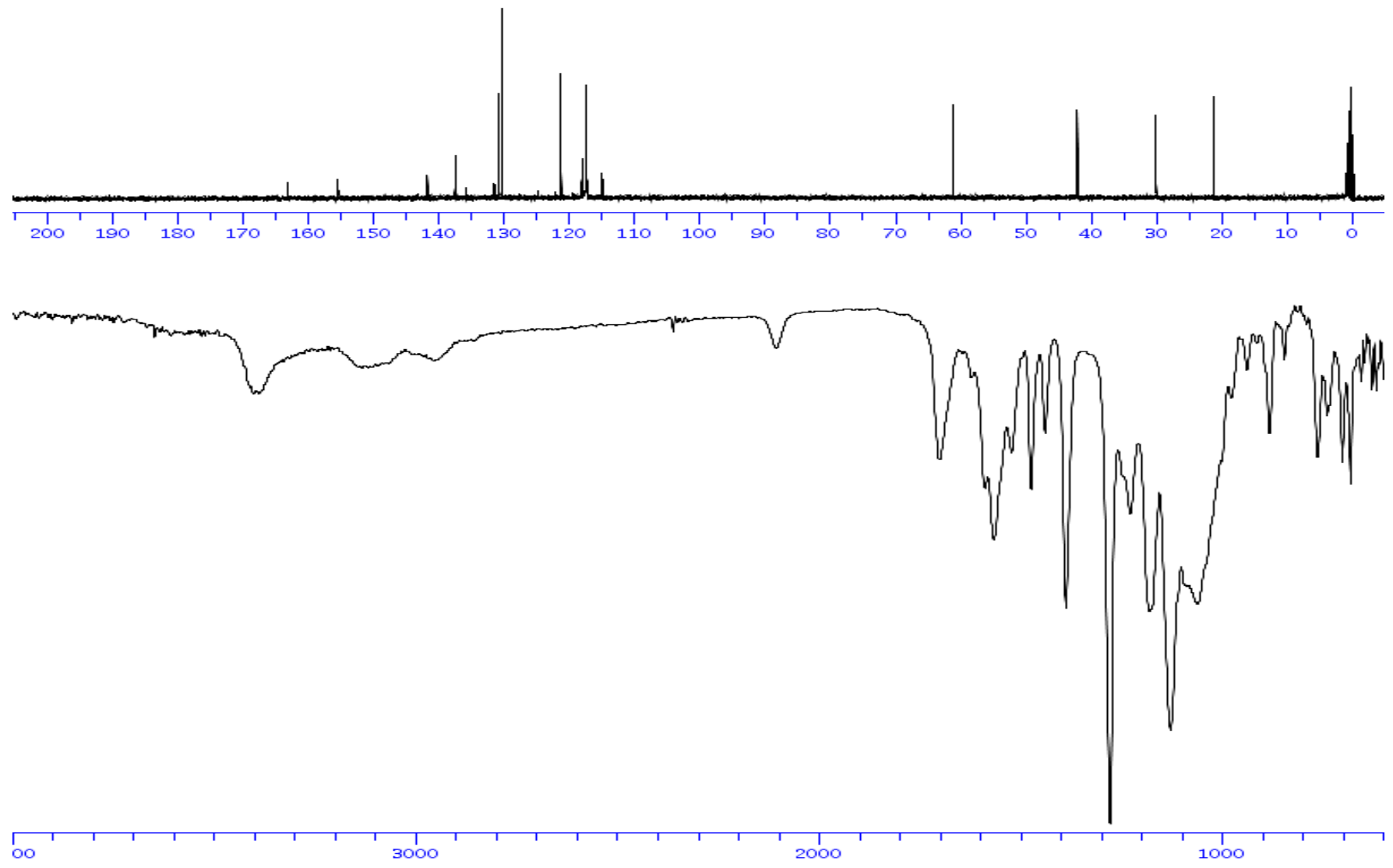


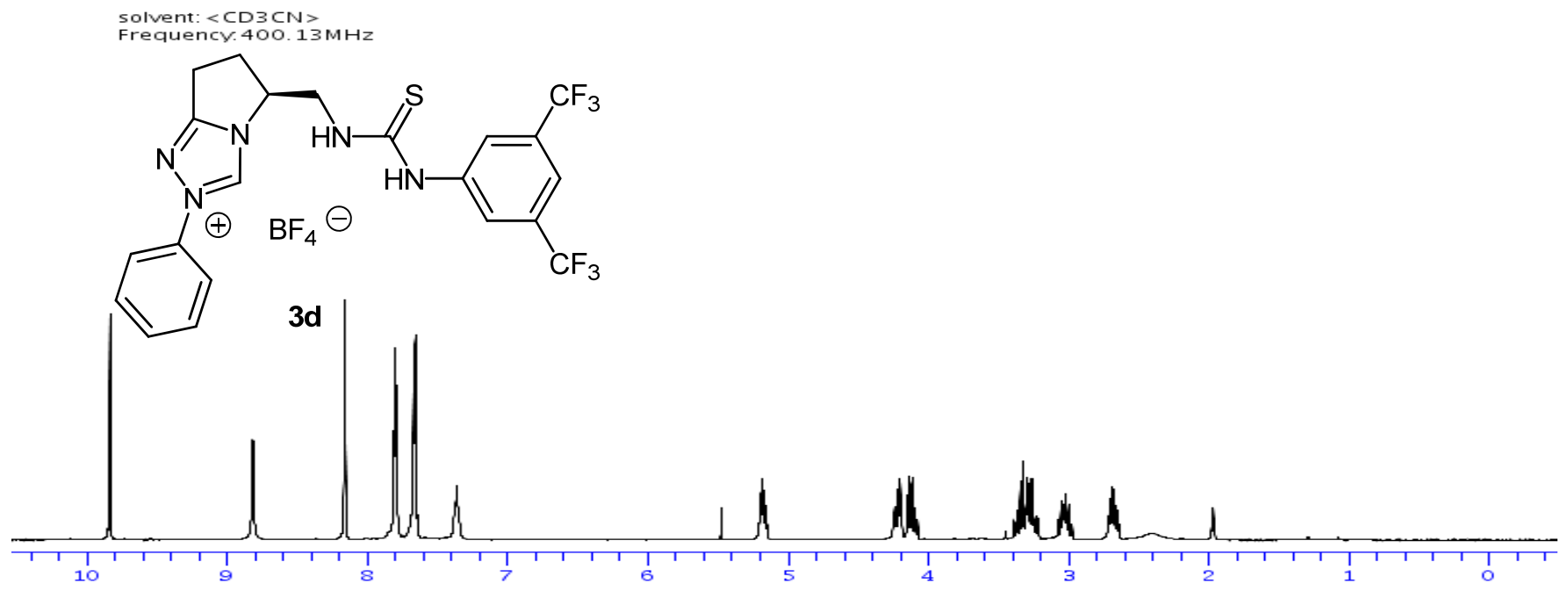

solvent: $\angle C D C I 3>$
Frequency. $100.612769 \mathrm{MHz}$

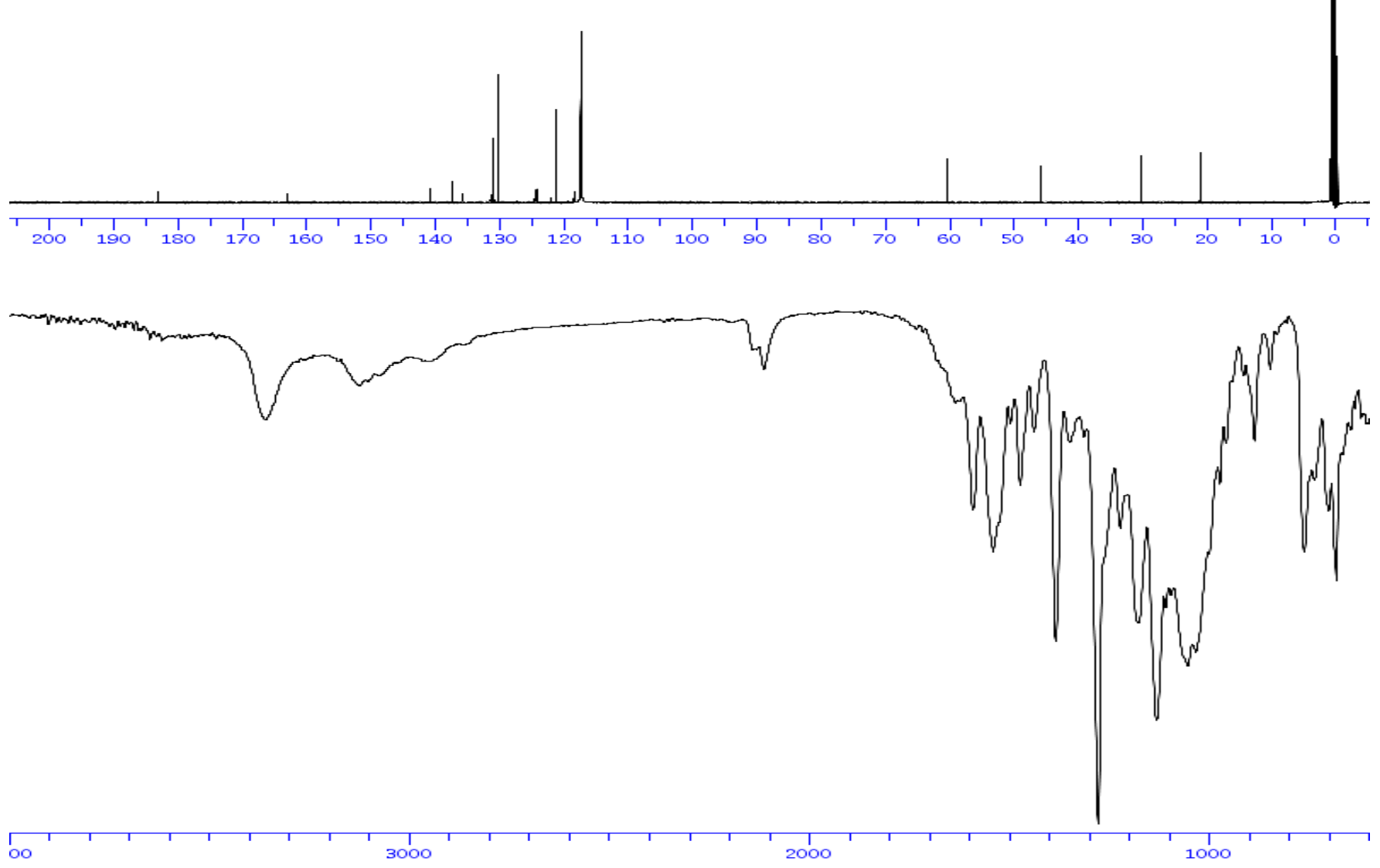

13 